\title{
Properties of the Boltzmann equation in the classical approximation
}

\author{
Thomas Epelbaum ${ }^{1}$, François Gelis ${ }^{1}$, Naoto Tanji ${ }^{2,3}$, Bin $\mathrm{Wu}^{1}$
}

August 6, 2018

1. Institut de Physique Théorique (URA 2306 du CNRS)

CEA/DSM/Saclay, 91191 Gif-sur-Yvette Cedex, France

2. Theoretical Research Division, Nishina Center, RIKEN

Wako 351-0198, Japan

3. Physics Department, Brookhaven National Laboratory

Upton, NY 11973, USA

\begin{abstract}
We study the Boltzmann equation with elastic point-like scalar interactions in two different versions of the the classical approximation. Although solving numerically the Boltzmann equation with the unapproximated collision term poses no problem, this allows one to study the effect of the ultraviolet cutoff in these approximations. This cutoff dependence in the classical approximations of the Boltzmann equation is closely related to the non-renormalizability of the classical statistical approximation of the underlying quantum field theory. The kinetic theory setup that we consider here allows one to study in a much simpler way the dependence on the ultraviolet cutoff, since one has also access to the non-approximated result for comparison.
\end{abstract}

\section{Introduction}

In the early stages of high energy heavy ion collisions, a dense gluonic matter is formed, nicknamed "glasma" [1]. At leading order in the strong coupling constant $g^{2}$, the evolution of these gluon fields is very simple, since it obeys the classical Yang-Mills equations [2]. However, at this order, it does not thermalize, nor does it exhibit the quasi-perfect hydrodynamical behavior that seems to be required by many bulk observables in RHIC and LHC data [3]. Next-to-leading order corrections to observables are quadratic in small perturbations around the LO classical gauge field $[4,5]$. However, due to the Weibel instability, these perturbations grow exponentially in time [6], and the NLO corrections can become larger than the LO. The resummation of an infinite class of higher 
order contributions, at all loop orders, is necessary in order to stabilize the perturbative expansion.

The Classical Statistical Approximation (CSA) is one of the schemes that has been employed recently in order to realize such a resummation $[7,8,9$, $10,11,12,13,14]$. Since it amounts to averaging solutions of the classical equations of motion over a Gaussian ensemble of initial conditions, its practical implementation is not more difficult than the LO calculation itself. Moreover, it trivially gives gauge invariant results for physical observables. By adjusting appropriately the 2-point correlation function that characterizes the Gaussian fluctuations of the initial classical fields, one can set up the CSA in such a way that it reproduces exactly the LO and NLO results, plus a subset of the higher order contributions $[15,16,17]$. By construction, the CSA is affected by the ultraviolet divergences that arise in loop corrections. For instance, all the divergences present at 1-loop are also present in the CSA since it is built precisely to reproduce exactly the NLO result. Practical implementations of the CSA represent space on a lattice, which provides a natural ultraviolet cutoff ${ }^{1}$.

It was observed in large scale lattice simulations using the CSA [14] that this approximation produces results that depend strongly on the ultraviolet cutoff when it is chosen to be much larger than the physical scales of the problem. In ref. [18], it was shown that the CSA is a non-renormalizable approximation of the underlying quantum field theory. The problem starts at 2-loops (the order where the CSA first differs from the underlying theory), and is due to the fact that the CSA includes some quantum corrections but not all of them ${ }^{2}$. Among the Feynman diagrams that contain non-renormalizable contributions, one can find some 2-loop self-energies that enter in the collision term of the Boltzmann equation, in the standard derivation of kinetic theory from the Dyson-Schwinger equations. This suggests that a similar cutoff dependence should be found in the classical approximation of the Boltzmann equation, and that one could investigate the ultraviolet artifacts of the CSA in the much simpler setting of kinetic theory. This would be particularly useful because the Boltzmann equation with $2 \rightarrow 2$ elastic scatterings is simple enough to be solved numerically without doing the classical approximation, thereby provided the "exact" result as a reference, something that cannot be done in the underlying quantum field theory ${ }^{3}$.

In this paper, we start from the classical approximation of the Boltzmann equation already discussed in refs. [22, 23], and we focus on issues related to the dependence of its solutions on the ultraviolet cutoff. In fact, we consider

\footnotetext{
${ }^{1}$ The loop momentum that causes the UV divergences is the momentum of the initial fluctuations. On the lattice, this momentum cannot exceed the inverse lattice spacing. However, it is also possible to limit by hand the largest momentum mode included in these initial fluctuations. When doing this, the effective UV regulator is no longer the inverse lattice spacing, but this cutoff that has been introduced in the spectrum of fluctuations.

${ }^{2}$ The CSA captures correctly the quantum corrections to the initial state, while the time evolution remains purely classical. Renormalizability requires that both types of quantum corrections be treated on the same footing.

${ }^{3} \mathrm{~A}$ similar kinetic treatment, although looking at a different problem, was recently performed in Yang-Mills theory [19, 20], using the Boltzmann equation derived in [21].
} 
two versions of the classical approximation, that correspond to including or not vacuum fluctuations in the underlying quantum field theory. We will compare the solutions of the unapproximated Boltzmann equation with the solutions obtained in these two approximations.

Our paper is organized as follows. In the section 2, we rederive the classical approximations of the Boltzmann equation, by following the same approach as in ref. [18] in order to emphasize the fact that the approximation is identical to the one that leads to the CSA. In the section 3, we generalize the Boltzmann equation in order to include the possibility of Bose-Einstein condensation. This is necessary if one wants to consider overoccupied initial conditions, as would be the matter formed in heavy ion collisions. In the section 4 , we first study the explicit dependence on the UV cutoff of the collision term in the classical approximation. In one version of this approximation, the collision term contains a piece which is quadratic in the UV cutoff, and is a direct consequence of the non-renormalizable contributions exhibited in ref. [18]. In the section 5, we study the fixed points of the Boltzmann equation in the two versions of the classical approximation, by extending to these modified equations the standard proof of the H-theorem. We also show that the fixed points in the classical approximation are strongly dependent on the UV cutoff, in a way which is in perfect agreement with the CSA computations of ref. [14]. Finally, in the section 6 , we compare the time evolutions in the classical approximations and in the unapproximated Boltzmann equation. The appendices contain some more technical material. The appendix A gives some details bout the calculation of the self-energies that enter in the expression of the collision term. In the appendix B, we remind the reader about the reduction of the collision term to a 2-dimensional integral (for an isotropic particle distribution), and we give some details about the discretization we use in order to compute it. In the appendix $\mathrm{C}$, we give explicit formulas for the spurious linear terms that appear in the classical collision term. In the appendix D, we give a brief discussion about how the existence of a Bose-Einstein condensate depends on the UV cutoff.

\section{Boltzmann eq. in the classical approximation}

\subsection{Collision term in the Schwinger-Keldysh formalism}

Our starting point will be the Boltzmann equation written in the following form,

$$
\left[\partial_{t}+\boldsymbol{v}_{\boldsymbol{p}} \cdot \boldsymbol{\nabla}\right] f(p)=\underbrace{\frac{i}{2 \omega_{\boldsymbol{p}}}\left[f(\boldsymbol{p}) \Sigma_{-+}(P)-(1+f(\boldsymbol{p})) \Sigma_{+-}(P)\right]}_{\mathcal{C}_{\boldsymbol{p}}[f]},
$$

where $\Sigma_{+-}$and $\Sigma_{-+}$are the self-energies in the Schwinger-Keldysh formalism [24, 25], evaluated for an on-shell momentum $P$. Although $f(\boldsymbol{p})$ is spacetime dependent, we do not write explicitly the time and space arguments in order to lighten the notations. The self-energies $\Sigma_{+-}$and $\Sigma_{-+}$that enter in 
the collision term can be calculated from the perturbative diagrammatic rules of the Schwinger-Keldysh formalism, in which the propagators read

$$
\begin{aligned}
G_{++}^{0}(p) & =\frac{i}{p^{2}-m^{2}+i \epsilon}+2 \pi f(\boldsymbol{p}) \delta\left(p^{2}-m^{2}\right) \\
G_{--}^{0}(p) & =\frac{-i}{p^{2}-m^{2}-i \epsilon}+2 \pi f(\boldsymbol{p}) \delta\left(p^{2}-m^{2}\right) \\
G_{+-}^{0}(p) & =2 \pi\left(\theta\left(-p^{0}\right)+f(\boldsymbol{p})\right) \delta\left(p^{2}-m^{2}\right) \\
G_{-+}^{0}(p) & =2 \pi\left(\theta\left(p^{0}\right)+f(\boldsymbol{p})\right) \delta\left(p^{2}-m^{2}\right)
\end{aligned}
$$

and where the vertices of type + read $\Gamma_{++++}=-i g^{2}$ while those of type - are $\Gamma_{----}=+i g^{2}$. The lowest order contribution to the collision term is a 2-loop self-energy, that corresponds to the elastic $2 \rightarrow 2$ scattering process. For the record, let us recall here the $2 \rightarrow 2$ contribution to the collision term for scalar particles,

$$
\begin{aligned}
C_{\boldsymbol{p}}[f]=\frac{g^{4}}{4 \omega_{\boldsymbol{p}}} \int_{\boldsymbol{p}^{\prime} \boldsymbol{k} \boldsymbol{k}^{\prime}}(2 \pi)^{4} & \delta\left(P+K-P^{\prime}-K^{\prime}\right) \\
\times & f\left(\boldsymbol{p}^{\prime}\right) f\left(\boldsymbol{k}^{\prime}\right)(1+f(\boldsymbol{p}))(1+f(\boldsymbol{k})) \\
& \left.-f(\boldsymbol{p}) f(\boldsymbol{k})\left(1+f\left(\boldsymbol{p}^{\prime}\right)\right)\left(1+f\left(\boldsymbol{k}^{\prime}\right)\right)\right],
\end{aligned}
$$

where for the sake of brevity we have denoted

$$
\int_{\boldsymbol{k}} \equiv \int \frac{\mathrm{d}^{3} \boldsymbol{k}}{(2 \pi)^{3} 2 \omega_{\boldsymbol{k}}}
$$

the invariant phase space integral for an on-shell particle.

\subsection{Collision term in the retarded-advanced basis}

In order to make the classical approximation more transparent, we follow the procedure used in refs. [22, 23, 18], that consists in going from the SchwingerKeldysh basis to the retarded-advanced basis [25, 26, 27]. We follow the notations of the section 2.3 of ref. [18], where the transformation matrix is defined $\mathrm{as}^{4}$

$$
\Omega_{\alpha \epsilon} \equiv\left(\begin{array}{cc}
1 & -1 \\
1 / 2 & 1 / 2
\end{array}\right)
$$

which leads to the following transformed propagators :

$\mathbb{G}_{\alpha \beta}^{0} \equiv \sum_{\epsilon, \epsilon^{\prime}= \pm} \Omega_{\alpha \epsilon} \Omega_{\beta \epsilon^{\prime}} G_{\epsilon \epsilon^{\prime}}^{0}=\left(\begin{array}{cc}0 & G_{A}^{0}=G_{++}^{0}-G_{-+}^{0} \\ G_{R}^{0}=G_{++}^{0}-G_{+-}^{0} & G_{S}^{0}=\frac{1}{2}\left(G_{++}^{0}+G_{--}^{0}\right)\end{array}\right)$

It is easy to check the self-energies are related by the following formula,

$$
\Sigma_{\epsilon \epsilon^{\prime}}=\Omega_{\alpha \epsilon} \Omega_{\beta \epsilon^{\prime}} \Sigma_{\alpha \beta},
$$

\footnotetext{
${ }^{4}$ Although in ref. [18], this transformation was introduced for the vacuum Feynman rules, the transformation matrix is in fact medium independent. It remains unchanged even if the system contains a particle distribution $f(\boldsymbol{p})$.
} 
where $\boldsymbol{\Sigma}_{\alpha \beta}$ denotes the self-energy in the retarded-advanced basis. In particular, we have

$$
\begin{aligned}
& \Sigma_{+-}=-\boldsymbol{\Sigma}_{11}+\frac{1}{2} \boldsymbol{\Sigma}_{12}-\frac{1}{2} \boldsymbol{\Sigma}_{21} \\
& \Sigma_{-+}=-\boldsymbol{\Sigma}_{11}-\frac{1}{2} \boldsymbol{\Sigma}_{12}+\frac{1}{2} \boldsymbol{\Sigma}_{21} .
\end{aligned}
$$

Therefore, the collision term is given as follows in terms of the rotated selfenergies :

$$
C_{\boldsymbol{p}}[f]=\frac{i}{2 \omega_{\boldsymbol{p}}}\left[\boldsymbol{\Sigma}_{11}(P)+\left(f(\boldsymbol{p})+\frac{1}{2}\right)\left(\boldsymbol{\Sigma}_{21}(P)-\boldsymbol{\Sigma}_{12}(P)\right)\right] .
$$

Note that $\boldsymbol{\Sigma}_{11}$ is purely imaginary, as well as the difference $\boldsymbol{\Sigma}_{21}(P)-\boldsymbol{\Sigma}_{12}(P)$, so that the collision term is real, as it should.

In the retarded-advanced basis, the bare propagators are

$$
\begin{aligned}
& G_{21}^{0}(p)=\frac{i}{\left(p^{0}+i \epsilon\right)^{2}-\boldsymbol{p}^{2}-m^{2}}, \quad G_{12}^{0}(p)=\frac{i}{\left(p^{0}-i \epsilon\right)^{2}-\boldsymbol{p}^{2}-m^{2}}, \\
& G_{22}^{0}(p)=2 \pi\left(\frac{1}{2}+f(\boldsymbol{p})\right) \delta\left(p^{2}-m^{2}\right),
\end{aligned}
$$

and the non-zero vertices are $\Gamma_{1222}=-i g^{2}$ and $\Gamma_{1112}=-i g^{2} / 4$.

\subsection{Collision term in the classical approximation}

From the collision term expressed in the form of eq. (9), it is easy to perform an approximation which is the exact analogue of the classical statistical approximation in the underlying quantum field theory. The Lagrangian of the theory in the retarded-advanced basis has two kinds of interaction terms, $\phi_{1} \phi_{2}^{3}$ and $\phi_{1}^{3} \phi_{2}$, where $2 \phi_{2}=\phi^{+}+\phi^{-}$and $\phi_{1}=\phi^{+}-\phi^{-}$. The classical approximation amounts to neglecting the $\phi_{1}^{3} \phi_{2}$ term. This limits the possible assignments for the indices 1 and 2 when constructing the graphs that contribute to the self-energies $\boldsymbol{\Sigma}_{12}$, $\boldsymbol{\Sigma}_{21}$ and $\boldsymbol{\Sigma}_{11}$. In the literature, there are in fact two versions of the classical approximation (that we will refer to as $\mathcal{C}^{0}$ and $\mathcal{C}^{1}$ thereafter) :

$\mathcal{C}^{0}$ : Neglect both the vertex $\Gamma_{1112}$ and the $1 / 2$ in the propagator $G_{22}^{0}$. This approximation coincides with the unapproximated theory at Leading Order in the coupling $g$ in the regime of strong fields $\left(\phi \sim g^{-1}\right)$ and/or large occupation numbers $\left(f(\boldsymbol{p}) \sim g^{-2}\right)$.

$\mathcal{C}^{1}$ : Neglect the vertex $\Gamma_{1112}$ but keep the $1 / 2$ in the propagator $G_{22}^{0}$. This approximation coincides with the unapproximated theory both at LO and NLO in the regime of strong fields $\left(\phi \sim g^{-1}\right)$ and/or large occupation numbers $\left(f(\boldsymbol{p}) \sim g^{-2}\right)$. 
The derivation of the collision term in these approximations is straightforward (see refs. [22, 23]) from the Feynman rules of the retarded-advanced formalism. If one starts from the full collision term (before doing the classical approximation), one can obtain the approximated collision terms by the following truncations and substitutions :

$\mathcal{C}^{0}$ : Keep only the terms of highest degree in $f$ in the collision term. For the $2 \rightarrow 2$ collision term, these terms are cubic in $f$.

$\mathcal{C}^{1}$ : Keep the terms of highest degree in $f$, and then substitute

$$
f \rightarrow f+\frac{1}{2} .
$$

For the elastic collision term, this gives the correct $f^{3}$ and $f^{2}$ terms, but also some spurious terms that are linear in $f$. More generally, for any contribution to the collision term, it can be shown that this ansatz gives all the terms with the leading and subleading powers in $f$.

Explicitly, the elastic collision term $\left(\boldsymbol{p} \boldsymbol{k} \leftrightarrow \boldsymbol{p}^{\prime} \boldsymbol{k}^{\prime}\right)$ in the $\mathcal{C}^{0}$ classical approximation reads

$$
\begin{aligned}
C_{\boldsymbol{p}}^{\mathcal{C}^{0}}[f] & =\frac{g^{4}}{4 \omega_{\boldsymbol{p}}} \int_{\boldsymbol{k}} \int_{\boldsymbol{p}^{\prime}} \int_{\boldsymbol{k}^{\prime}}(2 \pi)^{4} \delta\left(P+K-P^{\prime}-K^{\prime}\right) \\
\times & \left.\times f\left(\boldsymbol{p}^{\prime}\right) f\left(\boldsymbol{k}^{\prime}\right)(f(\boldsymbol{p})+f(\boldsymbol{k}))-f(\boldsymbol{p}) f(\boldsymbol{k})\left(f\left(\boldsymbol{p}^{\prime}\right)+f\left(\boldsymbol{k}^{\prime}\right)\right)\right] .
\end{aligned}
$$

For the $\mathcal{C}^{1}$ classical approximation, the elastic collision term reads

$$
\begin{aligned}
C_{\boldsymbol{p}}^{\mathcal{C}^{1}}[f] & =\frac{g^{4}}{4 \omega_{\boldsymbol{p}}} \int_{\boldsymbol{k}} \int_{\boldsymbol{p}^{\prime}} \int_{\boldsymbol{k}^{\prime}}(2 \pi)^{4} \delta\left(P+K-P^{\prime}-K^{\prime}\right) \\
\times & {\left[\left(f\left(\boldsymbol{p}^{\prime}\right)+\frac{1}{2}\right)\left(f\left(\boldsymbol{k}^{\prime}\right)+\frac{1}{2}\right)(1+f(\boldsymbol{p})+f(\boldsymbol{k}))\right.} \\
- & \left.\left(f(\boldsymbol{p})+\frac{1}{2}\right)\left(f(\boldsymbol{k})+\frac{1}{2}\right)\left(1+f\left(\boldsymbol{p}^{\prime}\right)+f\left(\boldsymbol{k}^{\prime}\right)\right)\right] .
\end{aligned}
$$

Our goal in this paper is to investigate the effect of replacing the unapproximated collision term $C_{\boldsymbol{p}}[f]$ by its classical approximations, given in eqs. (12) and (13), in order to gain some insight about the limitations of the classical statistical approximation, especially regarding its dependence on the ultraviolet cutoff.

\section{Boltzmann equation with a BEC}

If one takes into account only $2 \rightarrow 2$ processes, some initial conditions can have more particles than can be accommodated in a Bose-Einstein distribution ${ }^{5}$. At a given temperature, the maximal number of particles is achieved when the

\footnotetext{
${ }^{5}$ See $[28,29]$ for a similar discussion for a dense system of gluons in perturbative QCD.
} 
chemical potential is equal to the mass of the particles. If the initial condition contains more particles than this value, then the extra particles will form a Bose-Einstein condensate (BEC) at $\boldsymbol{p}=0^{6}$. To account for this possibility, let us replace the distribution $f(\boldsymbol{p})$ by

$$
f(\boldsymbol{p})+n_{c}(2 \pi)^{3} \delta(\boldsymbol{p}),
$$

with the understanding that the integral of $f(\boldsymbol{p})$ in a small sphere of radius $\epsilon$ around $\boldsymbol{p}=0$ goes to zero as $\epsilon \rightarrow 0$. This separation of the distribution into a continuous piece and a singular piece at the origin is very useful when solving numerically the Boltzmann equation, because of the impossibility to properly represent a delta function after discretizing momentum space.

After this separation, one gets coupled equations for the evolution of $f$ and $n_{c}$ [30]. Formally, these can be derived by injecting eq. (14) into the usual Boltzmann equation, and by using the delta function that comes with $n_{c}$ in order to simplify the integrals. It is important to note that terms that are at most linear in $n_{c}$ can emerge from the collision term; the terms in $n_{c}^{2}$ and $n_{c}^{3}$ all vanish because of kinematics or because the gain and loss terms cancel. If we start from the complete $2 \rightarrow 2$ collision term, we get the following two coupled equations

$$
\begin{aligned}
& \partial_{t} f(\boldsymbol{p})=\frac{g^{4}}{4 \omega_{\boldsymbol{p}}} \int_{\boldsymbol{p}^{\prime} \boldsymbol{k} \boldsymbol{k}^{\prime}}(2 \pi)^{4} \delta\left(P+K-P^{\prime}-K^{\prime}\right) \\
& \times \\
& \quad\left[f\left(\boldsymbol{p}^{\prime}\right) f\left(\boldsymbol{k}^{\prime}\right)(1+f(\boldsymbol{p}))(1+f(\boldsymbol{k}))\right. \\
&\left.-f(\boldsymbol{p}) f(\boldsymbol{k})\left(1+f\left(\boldsymbol{p}^{\prime}\right)\right)\left(1+f\left(\boldsymbol{k}^{\prime}\right)\right)\right] \\
&+\frac{g^{4} n_{c}}{8 m \omega_{\boldsymbol{p}}} \int_{\boldsymbol{p}^{\prime} \boldsymbol{k}^{\prime}}(2 \pi)^{4} \delta\left(\omega_{\boldsymbol{p}}+m-\omega_{\boldsymbol{p}^{\prime}}-\omega_{\boldsymbol{k}^{\prime}}\right) \delta\left(\boldsymbol{p}-\boldsymbol{p}^{\prime}-\boldsymbol{k}^{\prime}\right) \\
& \times {\left[f\left(\boldsymbol{p}^{\prime}\right) f\left(\boldsymbol{k}^{\prime}\right)(1+f(\boldsymbol{p}))\right.} \\
&\left.-f(\boldsymbol{p})\left(1+f\left(\boldsymbol{p}^{\prime}\right)\right)\left(1+f\left(\boldsymbol{k}^{\prime}\right)\right)\right] \\
&+\frac{g^{4} n_{c}}{4 m \omega_{\boldsymbol{p}}} \int_{\boldsymbol{k} \boldsymbol{k}^{\prime}}(2 \pi)^{4} \delta\left(\omega_{\boldsymbol{p}}+\omega_{\boldsymbol{k}}-m-\omega_{\boldsymbol{k}^{\prime}}\right) \delta\left(\boldsymbol{p}+\boldsymbol{k}-\boldsymbol{k}^{\prime}\right) \\
& \times\left[f\left(\boldsymbol{k}^{\prime}\right)(1+f(\boldsymbol{p}))(1+f(\boldsymbol{k}))\right. \\
&\left.-f(\boldsymbol{p}) f(\boldsymbol{k})\left(1+f\left(\boldsymbol{k}^{\prime}\right)\right)\right]
\end{aligned}
$$

and

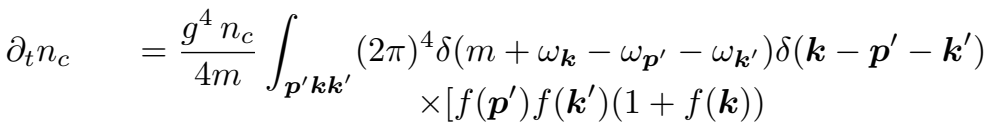

$$
\begin{aligned}
& \left.-f(\boldsymbol{k})\left(1+f\left(\boldsymbol{p}^{\prime}\right)\right)\left(1+f\left(\boldsymbol{k}^{\prime}\right)\right)\right] .
\end{aligned}
$$

It is straightforward to check that these coupled equations lead to the following

\footnotetext{
${ }^{6}$ If we assume that the system reaches an equilibrium made of a Bose-Einstein distribution with a chemical potential $\mu$, plus some extra particles of momentum $\boldsymbol{p}_{*}$, then it is possible to show from the Boltzmann equation that the only possibility to have a vanishing collision term is to have $\mu=m$ and $\boldsymbol{p}_{*}=0$.
} 
conservation laws :

$$
\begin{aligned}
n_{c}+\int \frac{d^{3} \boldsymbol{p}}{(2 \pi)^{3}} f(\boldsymbol{p}) & =\text { const } \\
m n_{c}+\int \frac{d^{3} \boldsymbol{p}}{(2 \pi)^{3}} \omega_{\boldsymbol{p}} f(\boldsymbol{p}) & =\text { const } \\
\int \frac{d^{3} \boldsymbol{p}}{(2 \pi)^{3}} \boldsymbol{p} f(\boldsymbol{p}) & =\text { const. }
\end{aligned}
$$

\section{Ultraviolet divergences in eq. (13)}

In this section, we extract the sources of explicit dependence on the ultraviolet cutoff in the classical approximations of the collision term. By explicit, we mean the UV cutoff dependence even if one assumes that the distribution $f(p)$ has a compact support and therefore does not extend up to the cutoff.

Firstly, let us note that there is no dependence on the UV cutoff coming from the terms that are cubic and quadratic in the distribution $f$ when $f(p)$ drops faster than $1 / p$ at large $p$. Therefore, the unapproximated collision term is UV finite. Likewise, the classical approximation given in eq. (12), that retains only the cubic terms, has an UV finite collision term. However, as we shall see later, the fixed point of the corresponding Boltzmann equation acquires a dependence on the UV cutoff due to the fact that $f(p)$ converges towards a distribution that falls like $1 / p$.

In the rest of this section, we will consider only the case of the second form of the classical approximation, in which the collision term is given by eq. (13).

\subsection{Kubo-Martin-Schwinger symmetry}

Let us first stress a subtlety in the derivation of the formula (13) from eq. (9). As one can see, eq. (13) vanishes if the particle distribution is identically zero. Physically, this is expected since this property means that the collision rate is zero when there are no particles in the system. Going back to eq. (9), this property is equivalent to the identity

$$
\left[\boldsymbol{\Sigma}_{11}(P)+\frac{1}{2}\left(\boldsymbol{\Sigma}_{21}(P)-\boldsymbol{\Sigma}_{12}(P)\right)\right]_{\text {vacuum }}=0 .
$$

This identity should be true because it is a consequence of the Kubo-MartinSchwinger [31, 32] (KMS) symmetry ${ }^{7}$.

It turns out that this identity is true in the classical statistical approximation $\mathcal{C}^{1}$ as well, but in a rather subtle way. Individually, $\boldsymbol{\Sigma}_{11}$ and $\boldsymbol{\Sigma}_{21}-\boldsymbol{\Sigma}_{12}$ are given by ultraviolet divergent integrals, and make sense only when regularized with

\footnotetext{
${ }^{7}$ In the Schwinger-Keldysh formalism, the KMS symmetry states that $\Sigma_{+-}(P)=$ $\exp \left(-p_{0} / T\right) \Sigma_{-+}(P)$ for a system in equilibrium at the temperature $T$. Taking the limit $T \rightarrow 0^{+}$and assuming that $p_{0}>0$, this leads to $\Sigma_{+-}^{\text {vacuum }}\left(p_{0}>0\right)=0$, which is equivalent to eq. (18).
} 
some cutoff $\Lambda_{\mathrm{UV}}$. When $P^{2}=0$ and $p_{0}>0$, we have (see the appendix A for some intermediate steps of this calculation)

$$
\operatorname{Im} \boldsymbol{\Sigma}_{11}(P)=\frac{I_{+}+I-}{6} \quad, \quad \operatorname{Im} \boldsymbol{\Sigma}_{21}(P)=\frac{I_{+}-I-}{2}
$$

where

$$
I_{+}=-\frac{g^{4}}{512 \pi^{3}}\left(\Lambda_{\mathrm{UV}}^{2}-\frac{2}{3} p^{2}\right) \quad, \quad I_{-}=-\frac{g^{4}}{256 \pi^{3}}\left(\Lambda_{\mathrm{UV}}^{2}-\frac{2}{3} p^{2}\right) .
$$

When combined into eq. (18), these quantities cancel and produce the expected identity. There is however a hidden complication: this cancellation works only provided that one does not break the symmetry between the three internal lines of the 2-loop self-energy. In deriving the eqs. (20), we have been careful to use a regularization that does not to introduce any asymmetry between the internal lines, but more naive regularizations will in general break this identity. This is something to keep in mind in numerical implementations of this approximation ${ }^{8}$.

\subsection{Terms quadratic in the UV cutoff}

Assuming that such a symmetric regularization has been employed, there is no $f$-independent term in the classical collision term. Since the terms that are cubic and quadratic in $f$ are UV finite, the potentially UV divergent part of the collision term comes from the terms that are linear in $f$,

$$
\begin{aligned}
C_{\boldsymbol{p}, \operatorname{lin}}^{\mathcal{C}^{1}}[f]=\frac{g^{4}}{16 \omega_{\boldsymbol{p}}} \int_{\boldsymbol{k}} \int_{\boldsymbol{p}^{\prime}} \int_{\boldsymbol{k}^{\prime}}(2 \pi)^{4} \delta\left(P+K-P^{\prime}-K^{\prime}\right) \\
\times\left[f\left(\boldsymbol{p}^{\prime}\right)+f\left(\boldsymbol{k}^{\prime}\right)-f(\boldsymbol{p})-f(\boldsymbol{k})\right] .
\end{aligned}
$$

From this formula, an explicit calculation shows that the Boltzmann equation in the classical approximation $\mathcal{C}^{1}$ contains a term which is quadratic in the UV cutoff (see the appendix $\mathrm{C}$ for the full expression of $C_{\boldsymbol{p}, \text { lin }}^{\mathcal{C}^{1}}[f]$ ),

$$
\left(\partial_{t}+\boldsymbol{v}_{\boldsymbol{p}} \cdot \nabla\right) f(\boldsymbol{p})=-\frac{g^{4} \Lambda_{\mathrm{UV}}^{2}}{1024 \pi^{3}} \frac{1}{\omega_{\boldsymbol{p}}} f(\boldsymbol{p})+\cdots
$$

where the dots are terms that have a lesser degree in the UV cutoff (including terms that are UV finite). Note that this term is a direct consequence of the term quadratic in $\Lambda_{\mathrm{UV}}$ contained in $\boldsymbol{\Sigma}_{21}-\boldsymbol{\Sigma}_{12}$, that was exhibited in ref. [18] and

\footnotetext{
${ }^{8}$ The main issue is with $\boldsymbol{\Sigma}_{12}$, that contains one retarded $G_{R}^{0}$ and two symmetric propagators $G_{S}^{0}$. In some numerical implementations of the classical statistical approximation, the spectrum of the initial fluctuations is cut at a scale $\Lambda_{\mathrm{UV}}$ which is smaller than the lattice ultraviolet cutoff. This amounts to limiting to $\Lambda_{\mathrm{UV}}$ the momentum flowing through the symmetric propagators, while the momentum flowing through the retarded propagators can go all the way up to the lattice cutoff. In these implementations, eq. (18) is not satisfied, and there is a non-zero collision rate even for an empty system.
} 
is a direct consequence of the non-renormalizability of the classical statistical approximation.

This term in $\Lambda_{\mathrm{UV}}^{2}$ introduces in the solution of this Boltzmann equation a contamination by cutoff effects, no matter what the initial $f(\boldsymbol{p})$ is. In other words, even with an initial $f(\boldsymbol{p})$ that has a compact support and therefore does not touch the UV cutoff, this term makes the solution immediately cutoff dependent (the magnitude of the contamination, and how quickly it affects the solution, of course depend on the coupling constant). If one keeps only this term and one assumes that the system is spatially homogeneous, the solution would read :

$$
f(t, \boldsymbol{p}) \approx f(0, \boldsymbol{p}) \exp \left[-\frac{g^{4} \Lambda_{\mathrm{UV}}^{2}}{1024 \pi^{3}} \frac{t}{\omega_{\boldsymbol{p}}}\right] .
$$

This formula indicates that the dependence on the UV cutoff leads to a rapid depletion of the distribution at small momentum. This can be tested by comparing this analytical formula with the numerical solution of the Boltzmann equation with the collision term (13), shown in the figure 1 at four different times shortly after the start of the evolution. In this plot, one sees a very good

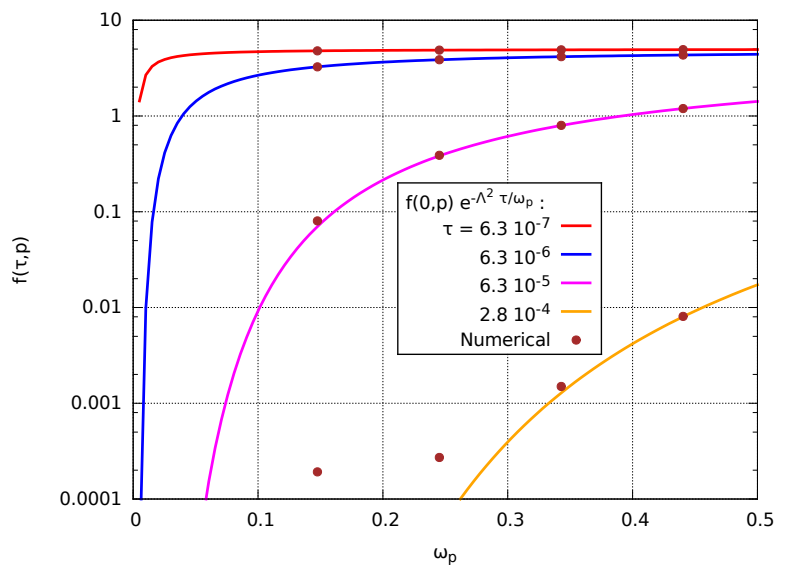

Figure 1: Evolution of $f(\boldsymbol{p})$ at short times in the classical approximation $\mathcal{C}^{1}$ for a large UV cutoff. Points : numerical resolution of eq. (13). Solid lines : right hand side of eq. (23) (we denote $\tau \equiv g^{4} t /\left(1024 \pi^{3}\right)$ ). Here, $\Lambda_{\mathrm{UV}}=100, f_{0}=5.0$, $Q=0.5$ and $m=0.05$.

agreement at short times between the analytical estimate and the numerical solution of the Boltzmann equation. It shows that, when the UV cutoff is large, its effects start at low momentum and grow exponentially in time. Note that the estimate of eq. (23) is accurate only at very small times (one can see deviations at the fourth of the times considered in the figure 1 ), because of the neglected subleading terms in the UV cutoff, and because of the terms in $f^{3}$ and 
$f^{2}$. At late times, when the solution approaches a fixed point, all the terms in the collision term are equally important and contribute to controlling the fixed point.

From the analytical solution given in eq. (23), one can estimate the timescale $t_{*}$ at which these spurious effects become corrections of order one,

$$
t_{*}^{-1} \sim \frac{g^{4} \Lambda_{\mathrm{UV}}^{2}}{1024 \pi^{3} Q},
$$

where $Q$ is the typical physical momentum scale. Equivalently, this can be written as

$$
Q t_{*} \sim \frac{1024 \pi^{3}}{g^{4}}\left(\frac{Q}{\Lambda_{\mathrm{UV}}}\right)^{2} .
$$

This timescale is reduced at larger couplings, or if the ultraviolet cutoff is taken far above the physical scale. Note however that the large numerical prefactor $1024 \pi^{3}$ helps to mitigate these effects, which suggests that $g=1$ is not a very strong coupling in this theory. In the following sections, we study the fixed points of the Boltzmann equation and the behavior of the solutions at large times, which means that we are well after this characteristic time. Our goal is to assess how strong is the dependence on the UV cutoff when $t \gtrsim t_{*}$.

\section{$5 \quad$ H-theorem and asymptotic state}

In the previous section, we have studied the explicit dependence on the UV cutoff of the collision term in the classical approximation. For distribution $f(\boldsymbol{p})$ with a compact support, we have seen that in the approximation $\mathcal{C}^{0}$ the collision term does not depend on $\Lambda_{\mathrm{UV}}$. This implies that at early times, the evolution of $f(\boldsymbol{p})$ is not affected by the cutoff (provided that the cutoff is large enough for the support of $f(\boldsymbol{p})$ to be below the cutoff). In contrast, in the approximation $\mathcal{C}^{1}$, the collision term contains a term which is quadratic in the cutoff. This term leads to a sensitivity of $f(\boldsymbol{p})$ on the cutoff even at early times, which is more prominent if the cutoff is larger and at small momentum $p$.

In this section, we now investigate the limit where time goes to infinity. In the Boltzmann equation, the allowed asymptotic states and the convergence of $f(\boldsymbol{p})$ to a solution of that form can be proven on the basis of conservation laws alone, and does not require any numerical study. By doing so for the two classical approximations considered in this paper, we will gain further insight on the effects of the classical approximation.

\section{$5.1 \quad$ H-theorem}

\subsubsection{Collisional invariants}

The first thing to note about eqs. (12) and (13) is that the approximations performed on the combinations of $f$ 's that appear in the collision term do not affect conservation laws, of particle number, energy and momentum. Moreover, 
the integrand in these approximate collision terms is still symmetric under the following exchanges,

$$
\begin{aligned}
& P \leftrightarrow K \\
& P^{\prime} \leftrightarrow K^{\prime}
\end{aligned}
$$

and antisymmetric under the exchange

$$
(P, K) \leftrightarrow\left(P^{\prime}, K^{\prime}\right)
$$

A collisional invariant $I(\boldsymbol{p})$ is a quantity (possibly momentum dependent) assigned to a particle, additive for an ensemble of particles, that is conserved in elementary collisions :

$$
I(\boldsymbol{p})+I(\boldsymbol{k})=I\left(\boldsymbol{p}^{\prime}\right)+I\left(\boldsymbol{k}^{\prime}\right) .
$$

Thanks to the above symmetries, it is easy to check that the total amount of this quantity in the system,

$$
\mathcal{I}[f] \equiv \int \frac{\mathrm{d}^{3} \boldsymbol{p}}{(2 \pi)^{3}} f(\boldsymbol{p}) I(\boldsymbol{p}),
$$

is constant in time if the particle distribution obeys the Boltzmann equation, regardless of whether we use the non-approximated collision term or the approximations of eqs. (12) or (13)

\subsubsection{Full quantum case}

For the non-approximated collision term, it is well known that one can define an entropy density,

$$
S_{\text {quantum }}=\int \frac{\mathrm{d}^{3} \boldsymbol{p}}{(2 \pi)^{3}}[(1+f(\boldsymbol{p})) \log (1+f(\boldsymbol{p}))-f(\boldsymbol{p}) \log (f(\boldsymbol{p}))]
$$

such that $\partial_{t} S_{\text {quantum }} \geq 0$ for any (positive definite) distribution ${ }^{9} f(\boldsymbol{p})$. Furthermore, $\partial_{t} S_{\text {quantum }}$ vanishes (i.e. entropy stops being produced) provided that $\log (f(\boldsymbol{p}) /(1+f(\boldsymbol{p})))$ is a linear combination of the collisional invariants allowed by the collision processes under consideration. For the elastic collisions considered here, these are the four components of the momentum, and the number of particles. This leads to the fact that the most general fixed point of the Boltzmann equation is a Bose-Einstein distribution with a chemical potential ${ }^{10}$.

\footnotetext{
${ }^{9}$ If the system is not spatially homogeneous, one must also introduce an entropy current $\boldsymbol{J}_{s}$, and the quantity which is always positive or zero is $\partial_{t} S_{\text {quantum }}+\boldsymbol{\nabla} \cdot \boldsymbol{J}_{s}$.

${ }^{10}$ If one also includes number changing processes in the collision term, the reasoning remains unchanged until the last step: the number of particles is no longer conserved and thus cannot appear in the equilibrium value of $\log (f(\boldsymbol{p}) /(1+f(\boldsymbol{p})))$. The allowed fixed points in this case are thus Bose-Einstein distributions with $\mu=0$.
} 


\subsubsection{Classical approximation $\mathcal{C}^{0}$}

This reasoning can be generalized to the Boltzmann equation in which the collision term has been approximated by eqs. (12) or (13), provided we modify the definition of the entropy density. For the collision term (12), the appropriate ${ }^{11}$ definition of the entropy density is

$$
S\left(\mathcal{C}^{0}\right)=\int \frac{\mathrm{d}^{3} \boldsymbol{p}}{(2 \pi)^{3}} \log (f(\boldsymbol{p})) .
$$

Using the Boltzmann equation, one finds that

$$
\begin{aligned}
\partial_{t} S\left(\mathcal{C}^{0}\right)=\frac{g^{4}}{2} & \int_{\boldsymbol{p}} \int_{\boldsymbol{k}} \int_{\boldsymbol{p}^{\prime}} \int_{\boldsymbol{k}^{\prime}}(2 \pi)^{4} \delta\left(P+K-P^{\prime}-K^{\prime}\right) \\
& \times \frac{1}{\alpha_{\boldsymbol{p}}}\left[\alpha_{\boldsymbol{p}^{\prime}} \alpha_{\boldsymbol{k}^{\prime}}\left(\alpha_{\boldsymbol{p}}+\alpha_{\boldsymbol{k}}\right)-\alpha_{\boldsymbol{p}} \alpha_{\boldsymbol{k}}\left(\alpha_{\boldsymbol{p}^{\prime}}+\alpha_{\boldsymbol{k}^{\prime}}\right)\right],
\end{aligned}
$$

where we denote $\alpha_{\boldsymbol{k}} \equiv f(\boldsymbol{k})$. Using the symmetries listed at the beginning of this subsection, this can be rewritten as

$$
\begin{aligned}
\partial_{t} S & \left(\mathcal{C}^{0}\right)=\frac{g^{4}}{8} \int_{\boldsymbol{p}} \int_{\boldsymbol{k}} \int_{\boldsymbol{p}^{\prime}} \int_{\boldsymbol{k}^{\prime}}(2 \pi)^{4} \delta\left(P+K-P^{\prime}-K^{\prime}\right) \\
& \times\left[\frac{1}{\alpha_{\boldsymbol{p}}}+\frac{1}{\alpha_{\boldsymbol{k}}}-\frac{1}{\alpha_{\boldsymbol{p}^{\prime}}}-\frac{1}{\alpha_{\boldsymbol{k}^{\prime}}}\right]\left[\alpha_{\boldsymbol{p}^{\prime}} \alpha_{\boldsymbol{k}^{\prime}}\left(\alpha_{\boldsymbol{p}}+\alpha_{\boldsymbol{k}}\right)-\alpha_{\boldsymbol{p}} \alpha_{\boldsymbol{k}}\left(\alpha_{\boldsymbol{p}^{\prime}}+\alpha_{\boldsymbol{k}^{\prime}}\right)\right]
\end{aligned}
$$

and it is now easy to check that the integrand is positive definite, which generalizes the $\mathrm{H}$-theorem to the classical statistical approximation $\mathcal{C}^{0}$ of the Boltzmann equation. Thanks to the factor

$$
\frac{1}{\alpha_{\boldsymbol{p}}}+\frac{1}{\alpha_{\boldsymbol{k}}}-\frac{1}{\alpha_{\boldsymbol{p}^{\prime}}}-\frac{1}{\alpha_{\boldsymbol{k}^{\prime}}}
$$

in the integrand, we also conclude that the fixed point is reached if $1 / \alpha_{\boldsymbol{p}}$ is a linear combination of collisional invariants, which for elastic collisions gives the following most general equilibrium distribution,

$$
f_{\mathcal{C}^{0}}(\boldsymbol{p})=\frac{T}{\omega_{\boldsymbol{p}}-\mu}
$$

Note that this expression is made of the first term in the expansion of the BoseEinstein distribution in the limit of small $\omega_{\boldsymbol{p}}$. Unlike the full Bose-Einstein distribution that has an exponentially falling tail, this equilibrium distribution decreases only as a power law. As we will see in the next section, when inserted into the equations of particle number and energy conservation, this leads to parameters $T$ and $\mu$ that depend on the UV cutoff.

\footnotetext{
${ }^{11}$ What is "appropriate" is judged by whether the definition agrees with eq. (28) at large $f$ (to LO in the case of eq. (12), and to LO and NLO in the case of eq. (13)), and whether it leads to a monotonously increasing entropy with time.
} 


\subsubsection{Classical approximation $\mathcal{C}^{1}$}

In the case of the collision term (13), one should use

$$
S\left(\mathcal{C}^{1}\right)=\int \frac{\mathrm{d}^{3} \boldsymbol{p}}{(2 \pi)^{3}} \log \left(\frac{1}{2}+f(\boldsymbol{p})\right) .
$$

The reasoning is exactly the same as in the case of the approximation $\mathcal{C}^{0}$, and the equations (30) and (31) remain valid for the derivative $\partial_{t} S\left(\mathcal{C}^{1}\right)$ provided that we now define $\alpha_{\boldsymbol{k}} \equiv f(\boldsymbol{k})+\frac{1}{2}$. The fixed points are still obtained by imposing that $1 / \alpha_{\boldsymbol{p}}$ be a linear combination of collisional invariants. This gives :

$$
f_{\mathcal{C}^{1}}(\boldsymbol{p})=\frac{T}{\omega_{\boldsymbol{p}}-\mu}-\frac{1}{2},
$$

which now corresponds to the first two orders in the expansion of the BoseEinstein distribution at small energy.

To conclude this subsection, let us note that when one includes only the $2 \rightarrow 2$ scattering in the collision term, all the coupling dependence factorizes in the factor $g^{4}$ that appears in the right hand side of the Boltzmann equation. One can therefore absorb this factor into a rescaling of the time axis, which means that the only effect of changing the coupling is to make the time evolution faster or slower (timescales are proportional to $g^{-4}$ ). But the outcome of the evolution at $t \rightarrow \infty$ does not depend on the value of the coupling, nor the above conclusion that the particle distribution will be driven eventually towards the functional form of eqs. (33) or (35), depending of which version of the classical approximation is used.

\subsection{Dependence of the fixed point on the UV cutoff}

The fixed point of the Boltzmann equation has a number of free parameters equal to the number of conserved quantities in the system, and therefore it is sufficient to write these conservation equations in order to determine these parameters. In the case of elastic collisions, in the classical approximation $\mathcal{C}^{1}$, $T$ and $\mu$ are related to the particle density and the energy density,

$$
\begin{aligned}
n & =n_{c}+\int \frac{\mathrm{d}^{3} \boldsymbol{p}}{(2 \pi)^{3}}\left(\frac{T}{\omega_{\boldsymbol{p}}-\mu}-\frac{1}{2}\right) \\
\epsilon & =n_{c} m+\int \frac{\mathrm{d}^{3} \boldsymbol{p}}{(2 \pi)^{3}}\left(\frac{T}{\omega_{\boldsymbol{p}}-\mu}-\frac{1}{2}\right) \omega_{\boldsymbol{p}} .
\end{aligned}
$$

Note that the chemical potential must be less than (or equal to) the mass of the particles for these integrals to be defined in the infrared. In these equations, the constant $n_{c}$ accounts for the possibility to form a Bose-Einstein condensate if the system is overoccupied.

The main issue with these equations is that the integrals in both of them are severely ultraviolet divergent. Let us therefore introduce an ultraviolet cutoff 
such that $|\boldsymbol{p}| \leq \Lambda_{U V}$; the integrals are now finite but depend also on $\Lambda_{U V}$, which implies that $T, \mu$ and $n_{c}$ will not only depend on the input quantities $n$ and $\epsilon$, but also on the cutoff. In fact, if $\Lambda_{U V}$ is much larger than the physical scales, then the values of $T$ and $\mu$ are completely dominated by the cutoff. This property of the Boltzmann equation in the classical approximation is closely related to the fact that the underlying field theory in this approximation is not renormalizable [18].

Let us nevertheless use eqs. (36) with an ultraviolet cutoff $\Lambda_{U V}$. They can be rewritten as

$$
\begin{aligned}
2 \pi^{2}\left(\widetilde{n}-\widetilde{n}_{c}\right)+\frac{1}{6} & =\widetilde{T} \int_{0}^{1} \mathrm{~d} x \frac{x^{2}}{\sqrt{x^{2}+\widetilde{m}^{2}}-\widetilde{\mu}} \\
2 \pi^{2}\left(\widetilde{\epsilon}-\widetilde{n}_{c} \widetilde{m}\right)+f(\widetilde{m}) & =\widetilde{T} \int_{0}^{1} \mathrm{~d} x \frac{x^{2} \sqrt{x^{2}+\widetilde{m}^{2}}}{\sqrt{x^{2}+\widetilde{m}^{2}}-\widetilde{\mu}},
\end{aligned}
$$

where we denote

$$
f(\widetilde{m}) \equiv \frac{1}{8}\left(\sqrt{1+\widetilde{m}^{2}}\left(1+\frac{\widetilde{m}^{2}}{2}\right)-\frac{\widetilde{m}^{4}}{2} \sinh ^{-1} \frac{1}{\widetilde{m}}\right)
$$

and where, for any quantity $X$ with mass dimension $d$, we denote $\widetilde{X} \equiv X / \Lambda_{U V}^{d}$. These equations define the asymptotic values ${ }^{12} \widetilde{T}, \widetilde{\mu}$ and $\widetilde{n}_{c}$ as a function of $\widetilde{\epsilon}$, $\widetilde{n}$ and $\tilde{m}$. If the cutoff is large compared to the physical momentum scale, then we have

$$
\widetilde{\epsilon}, \widetilde{n}, \widetilde{m} \ll 1 .
$$

Neglecting $\widetilde{m} \ll 1$ in eqs. (37), these equations become

$$
\begin{aligned}
2 \pi^{2}\left(\widetilde{n}-\widetilde{n}_{c}\right)+\frac{1}{6} & =\widetilde{T}\left[\frac{1}{2}+\widetilde{\mu}+\widetilde{\mu}^{2} \ln \left(\frac{1-\widetilde{\mu}}{-\widetilde{\mu}}\right)\right] \\
2 \pi^{2} \widetilde{\epsilon}+\frac{1}{8} & =\widetilde{T}\left[\frac{1}{3}+\frac{\widetilde{\mu}}{2}+\widetilde{\mu}^{2}+\widetilde{\mu}^{3} \ln \left(\frac{1-\widetilde{\mu}}{-\widetilde{\mu}}\right)\right],
\end{aligned}
$$

and for $\widetilde{\epsilon}, \widetilde{n} \ll 1$ their solutions are approximately given by

$$
\widetilde{n}_{c}=0 \quad, \quad \widetilde{\mu} \approx \frac{1}{80 \pi^{2}(4 \widetilde{\epsilon}-3 \widetilde{n})} \rightarrow-\infty \quad, \quad \widetilde{T} \approx-\frac{\widetilde{\mu}}{2} \rightarrow+\infty .
$$

Note that these values go to infinity very quickly if the ultraviolet cutoff $\Lambda_{U V}$ goes to infinity at fixed $\epsilon$ and $n$ (asymptotically, $\mu, T \sim \Lambda_{U V}^{4}$ ). This behavior of the asymptotic $T$ and $\mu$ is quite consistent with the observation made in the figure 10 of Ref. [14], although there the classical statistical approximation for the underlying field theory was used instead of the classical approximation of the Boltzmann equation. At finite ultraviolet cutoff, the eqs. (37) can be solved numerically, as illustrated in the figure 2. As one can see on this figure,

\footnotetext{
${ }^{12}$ It may seem at first sights that there are three unknowns $\left(\widetilde{T}, \widetilde{\mu}\right.$ and $\left.\widetilde{n}_{c}\right)$ for only two equations. However, this is not the case. If the system is not overoccupied, then $\widetilde{n}_{c}=0$ and there are only two unknowns. If the system is overoccupied, then we know a priori that $\widetilde{\mu}=\widetilde{m}$, and again only two of these parameters are truly unknown. A discussion of the condition for the existence of a Bose-Einstein condensate is presented in the appendix D.
} 


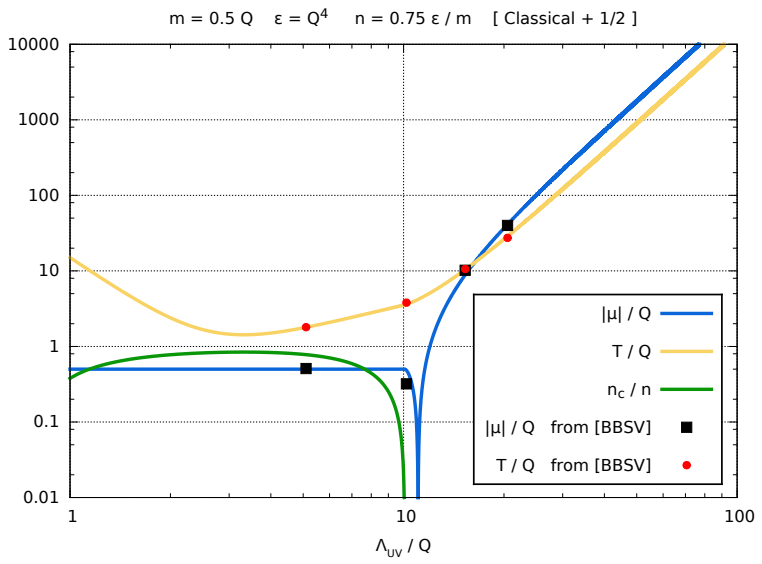

Figure 2: Evolution of the classical equilibrium parameters $T, \mu, n_{c}$ as a function of the ultraviolet cutoff, for the classical approximation $\mathcal{C}^{1}$. All the dimensionful quantities in the system are set in terms of a unique parameter $Q=\epsilon^{1 / 4}$ : in this example, $m=0.5 \cdot Q$ and $n=0.75 \cdot \epsilon / m . \mu$ is positive at small $\Lambda_{U V}$ and negative at large $\Lambda_{U V}$. The points reproduce the values listed in the figure 10 of Ref. [14], obtained with a classical statistical field simulation on a $768^{3}$ lattice.

the values of the equilibrium parameters $T, \mu$ and $n_{c}$ depend on the ultraviolet cutoff, especially strongly at large values of this cutoff. Even the conclusion regarding the existence of a Bose-Einstein condensate is cutoff dependent, since the condensate density $n_{c}$ vanishes above a certain value of the cutoff. When the cutoff is too small, some modes that are important in the dynamics of the system are excluded from the description. On the contrary, when the cutoff is large, the parameters of the fixed point are controlled by the ultraviolet divergence in the integrals of eqs. (36). Moreover, because the fixed point of the Boltzmann equation is independent of the value of the coupling, the system will eventually evolve towards these unphysical values no matter how small the coupling is. The only effect of a smaller coupling is to make the convergence towards this asymptotic state slower. Note also that there seem to be a "sweet spot", in the range $3 \lesssim \Lambda_{U V} / Q \lesssim 6$, where these parameters are the least sensitive to the value of the cutoff.

In the figure 2, we also show the comparison with the values of $T, \mu$ fitted in Ref. [14] from several classical statistical simulations with various ultraviolet cutoffs. The remarkable agreement ${ }^{13}$ with the asymptotic behavior of the elas-

\footnotetext{
${ }^{13}$ It is important to keep in mind that the full fledged classical statistical simulations contain some effects of inelastic processes, in contrast to the Boltzmann equation studied here. Therefore, in the limit of extremely large times, these simulations should always lead to a vanishing chemical potential. However, on the timescales considered in Ref. [14], this is not yet the case because they are still small compared to the inverse of the inelastic scattering
} 
tic Boltzmann equation in the classical approximation suggests that this much simpler equation is a very good model in order to perform studies of the range of validity of the classical statistical approximation. There are two advantages in using the Boltzmann equation for this purpose: (i) it is simpler to solve that the classical statistical field simulation ${ }^{14}$ and (ii) one can compute the solutions with and without the classical approximation equally easily. Given this, our goal in the rest of this paper is to address the following questions:

- Compare the full Boltzmann equation and the Boltzmann equation in the classical statistical approximation, by solving them numerically. Since changes of the coupling can be absorbed into a rescaling of time, we will do this at a unique coupling $g^{2}=1$.

- Assess how the value of the ultraviolet cutoff influences the results in the classical statistical approximation. In particular, is there a range of values of the cutoff that helps to reduce the differences between the full and approximated Boltzmann equations?

Before going into this, let us also show how the parameters of the fixed point depend on the UV cutoff in the case of the classical approximation $\mathcal{C}^{0}$. In this case, the two conservation equations lead to

$$
\begin{aligned}
n & =n_{c}+\int \frac{\mathrm{d}^{3} \boldsymbol{p}}{(2 \pi)^{3}}\left(\frac{T}{\omega_{\boldsymbol{p}}-\mu}\right) \\
\epsilon & =n_{c} m+\int \frac{\mathrm{d}^{3} \boldsymbol{p}}{(2 \pi)^{3}}\left(\frac{T}{\omega_{\boldsymbol{p}}-\mu}\right) \omega_{\boldsymbol{p}},
\end{aligned}
$$

which can easily be solved numerically. For the same set of input parameters as in the figure 2, one now finds the results of the figure 3 . In this case, the temperature drops to zero as $\Lambda_{\mathrm{UV}}^{-3}$ when the cutoff becomes large, while the chemical potential always goes to $\mu=m$. In this approximation, all the particles go into a Bose-Einstein condensate in the limit $\Lambda_{\mathrm{UV}} \rightarrow \infty$. This version of the classical approximation is rather subtle because, as we have mentioned before, the collision term is independent of $\Lambda_{\mathrm{UV}}$ provided that the distribution $f(\boldsymbol{p})$ has a compact support that does not extend to the cutoff. Therefore, it seems that for a sufficiently large cutoff, the solution should not depend on the cutoff. However, in this classical approximation, the limits $\Lambda_{\mathrm{UV}} \rightarrow \infty$ and $t \rightarrow \infty$ do not commute. The figure 3 amounts to taking the limit $t \rightarrow \infty$ first, and to look at the behavior of the resulting equilibrium parameters for a large $\Lambda_{\mathrm{Uv}}$.

rate.

${ }^{14} \mathrm{As}$ recalled in the appendix B, the collision term for elastic collisions in $\phi^{4}$ theory can be reduced to a 2-dimensional integral if the distribution is isotropic in momentum space. 


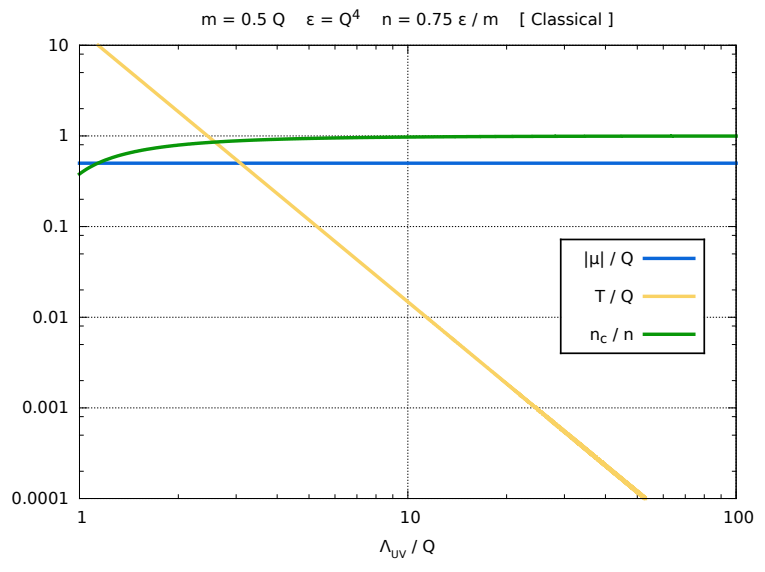

Figure 3: Evolution of the classical equilibrium parameters $T, \mu, n_{c}$ as a function of the ultraviolet cutoff, for the classical approximation $\mathcal{C}^{0}$. The setup is the same as in the figure 2 .

\section{Numerical study of the Boltzmann equation}

\subsection{Setup}

In order to go beyond the simple estimates and considerations based on conservation laws that we have performed in the previous sections, we now solve numerically the Boltzmann equation, in order to have access to the details of the time evolution. In order to do so, we must do some simplifying assumptions. Firstly, we assume that the system is spatially homogeneous in order to drop the $\boldsymbol{x}$ dependence in the distribution function. After this simplification, the collision term is still given by a 5-dimensional integral (after having taken into account energy and momentum conservation), which is still too computationally expensive. Therefore, we further assume that the distribution is isotropic in momentum space, which allows a drastic simplification of the collision term: all the angular integrations can be performed analytically, and the 5-dimensional integral is reduced to a 2-dimensional integral, which is now doable numerically. This reduction is well known ${ }^{15}$, but we reproduce it in the appendix B for the sake of completeness.

When solving numerically the Boltzmann equation, special care must be taken to satisfy with high accuracy the conservation of particle number, energy and momentum. This is particularly important in the vicinity of a Bose-Einstein condensation transition, where the system evolves very rapidly and where these

\footnotetext{
${ }^{15}$ Note that this simplification is specific to point-like scalar interactions. For more general scatterings, the matrix element may have a non-trivial angular dependence, preventing this analytical simplification.
} 
errors may grow in an uncontrollable manner. In the appendix B, we describe a discretization scheme with which these conservation laws remain exact (and thus are only limited by rounding errors in practice).

In this study, we are interested in initial distributions that are large for momenta below a certain characteristic scale $Q$, and negligible above $Q$. For simplicity, we take an initial distribution of the form

$$
f(0, p)=f_{0} \theta(Q-p) .
$$

$Q$ is not a true parameter of the problem, since all the dimensionful quantities can be expressed in units of $Q$. Likewise, the coupling constant appears only as a $g^{4}$ prefactor in the collision term, and its sole effect is to stretch or squeeze the timescales. We have therefore taken $g=1$ in all the numerical calculations. Starting with the same initial condition, we will compare the unapproximated Boltzmann equation with the classical approximations $\mathcal{C}^{0}$ and $\mathcal{C}^{1}$, for several values of the ratio $\Lambda_{\mathrm{Uv}} / Q$.

If $f_{0}$ is large enough, the system becomes overoccupied, which leads to the formation of a Bose-Einstein condensate. If one just solves the plain Boltzmann equation (with or without the classical approximation in the collision term), the solution becomes unstable at the time where the BEC transition would normally happen. In order to be able to pursue the numerical evolution beyond this transition, it is necessary to explicitly allow the presence of a condensate by solving the coupled equations described in the section 3 . When doing so, the region of the transition remains difficult to handle numerically, because the density of condensed particles grows by several orders of magnitude in a very short time. A very small timestep is necessary in this region. In order to avoid complications in the equations (16) and (17), we consider massive particles (in the numerical results presented later in this section, we have used $m=0.1 Q$ ).

From eq. (17), it is obvious that the initial value of $n_{c}$ must be non-zero for $n_{c}$ to have an effect on the system, since its evolution equation is homogeneous. However, the precise value of this initial condition is not very important, as long as $n_{c}(0)$ is negligible compared to the total particle density. This is illustrated in the figure 4 , where we have varied the initial value $n_{c}(0)$ by many orders of magnitude, at fixed $f_{0}=5$. For a small enough $n_{c}(0)$, the time at which the BEC transition occurs and the subsequent evolution of $n_{c}(t)$ do not depend on this initial value. In the rest of the paper, all the computations are done with the initial value $n_{c}(0)=10^{-5}$.

In the figure 5 , we show how the time evolution of the fraction of condensed particles $n_{c}(t) / n_{\text {total }}$ changes with the magnitude $f_{0}$ of the initial particle distribution. The behavior of this ratio is quite sensitive to the value of $f_{0}$, and a BEC transition happens for any $f_{0}$ larger than some critical value that one can estimate to be $f_{0}^{*} \approx 0.30484$. Moreover, by increasing $f_{0}$, the transition happens at earlier times, while the asymptotic value of the condensed fraction increases only slightly ${ }^{16}$. A more thorough description of the BEC transition in

\footnotetext{
${ }^{16}$ Increasing $f_{0}$ is not an efficient way to increase the condensate fraction. Indeed, by spreading the extra particles uniformly at all momenta from 0 to $Q$, one is mostly heating up the system.
} 


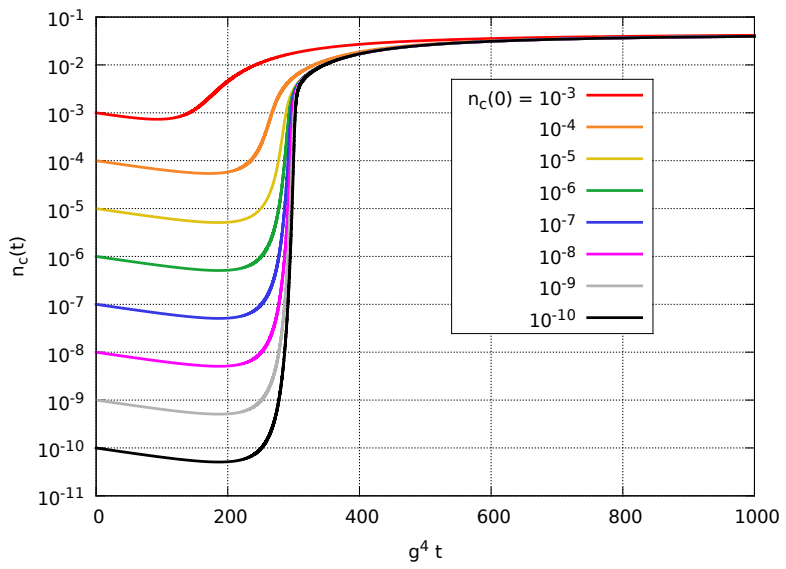

Figure 4: Time evolution of the density $n_{c}$ of condensed particles with the unapproximated collision term, for $n_{c}(0)$ varying from $10^{-3}$ to $10^{-10}$. The UV cutoff was set to $\Lambda_{\mathrm{UV}} / Q=4$, and $f_{0}=5$.

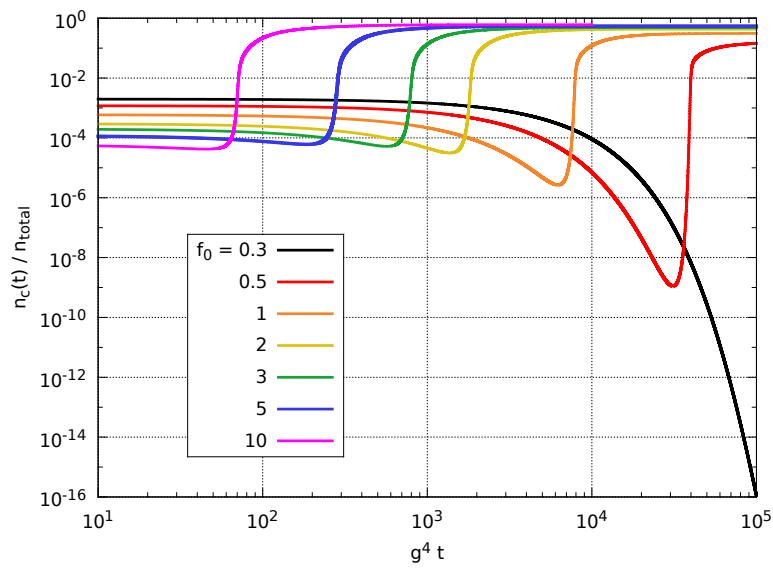

Figure 5: Time evolution of the fraction of particles in the zero mode, $n_{c}(t) / n_{\text {total }}$, with the unapproximated collision term, for $f_{0}=$ $0.3,0.5,1,2,3,5,10$. The UV cutoff was set to $\Lambda_{\mathrm{UV}} / Q=4$. 
kinetic theory can be found in refs. [33, 34, 30].

\subsection{Evolution of the condensate density}

Firstly, let us consider as a reference the case where we do not perform any classical approximation on the collision term. The initial condition we have used is of the form given in eq. (43), with $f_{0}=10$. In this case, the solution

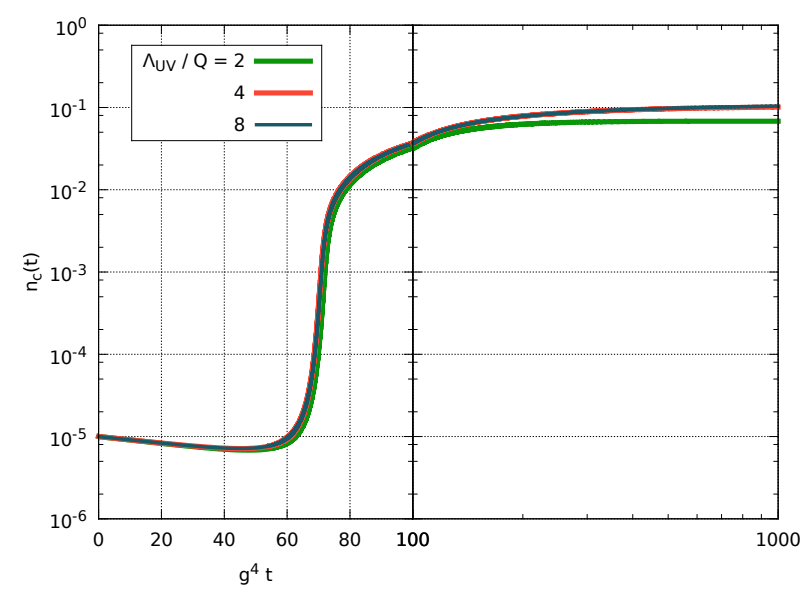

Figure 6: Time evolution of the density $n_{c}$ of condensed particles with the unapproximated collision term, for three values $\Lambda_{\mathrm{UV}} / Q=2,4$ and 8 of the UV cutoff.

of the Boltzmann equation has a well defined $\Lambda_{\mathrm{UV}} \rightarrow \infty$ limit, and the only requirement to observe this limiting behavior is to make the UV cutoff large enough compared to the physical scale. In the figure 6 , we illustrate this by showing the time dependence of the density $n_{c}$ of condensed particles (for an overoccupied initial condition), for three values $\Lambda_{\mathrm{UV}} / Q=2,4$ and 8 of the UV cutoff. We observe that the asymptotic value of $n_{c}$ changes a bit when going from $\Lambda_{\mathrm{UV}} / Q=2$ to 4 , but remains unchanged when increasing it further to 8 . This is the sign that the UV limit is already reached for values of the cutoff above $4 Q$. In the following, we will use this result as a reference, and refer to it as the "exact" result.

Consider now the classical approximation $\mathcal{C}^{0}$ to the collision term, in which one keeps only the cubic terms in the distribution function. The time evolution of the density of condensed particles is shown in the figure 7, for the same initial condition and the same values of the UV cutoff. In this approximation, $n_{c}(t)$ behaves in a very similar way as with the unapproximated collision term: the BEC transition happens at the same time, and the asymptotic density of condensed particles is approximately the same ${ }^{17}$.

${ }^{17}$ This aspect of the comparison is a bit misleading, and is true here only because we have 


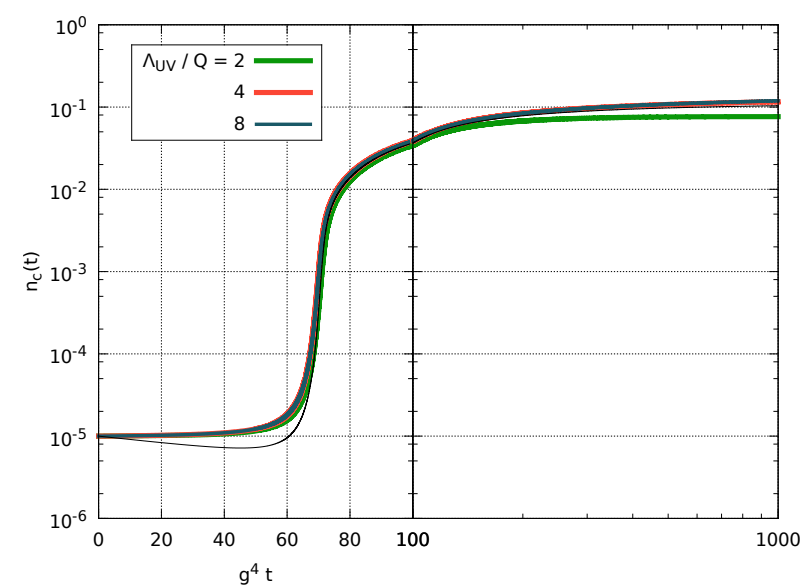

Figure 7: Time evolution of the density $n_{c}$ of condensed particles with the collision term in the classical approximation $\mathcal{C}^{0}$, for three values $\Lambda_{\mathrm{UV}} / Q=2,4$ and 8 of the UV cutoff. The thin black line is the solution of the unapproximated Boltzmann equation with $\Lambda_{\mathrm{UV}} / Q=8$.

In contrast, the classical approximation $\mathcal{C}^{1}$ (see the figure 8) tends to underestimate the density of particles in the condensate. When the UV cutoff is moderately above the physical scale $\left(\Lambda_{\mathrm{UV}} / Q=2,4\right.$ in the figure), the behavior of $n_{c}(t)$ in this approximation remains in qualitatively good agreement with the exact result (thin black line). However, for $\Lambda_{\mathrm{UV}} / Q=8$, the condensate is completely depleted when time goes to infinity. This is a more dynamical view of what we had learned in the previous section from the study of conservation laws. At large $\Lambda_{\mathrm{UV}} / Q$, the equilibrium chemical potential is always negative in this approximation and $n_{c}(t=\infty)=0$.

\subsection{Evolution of the distribution function}

In the previous subsection, we have seen that the initial condition (43) with $f_{0}=10$ leads to the formation of a Bose-Einstein condensate, at a time $g^{4} t \approx 70$. If the UV cutoff is not too large compared to the physical scale $Q$, both classical approximations reproduce qualitatively this behavior: the condensation time is almost the same, but the density of condensed particles differs a bit from the exact value (overestimated in the approximation $\mathcal{C}^{0}$ and underestimated in the approximation $\mathcal{C}^{1}$ ). Let us now continue this comparison by looking at the particle distribution itself, at various times. We do this for a moderate value

used a strongly overoccupied initial condition, for which a large fraction of the total number of particles condense in the zero mode. Indeed, the classical approximation $\mathcal{C}^{0}$, that always tends to put all the particles in the condensate when $\Lambda_{\mathrm{UV}} \rightarrow \infty$ (see the previous section), is bound to give a similar $n_{c}$ in such a situation. 


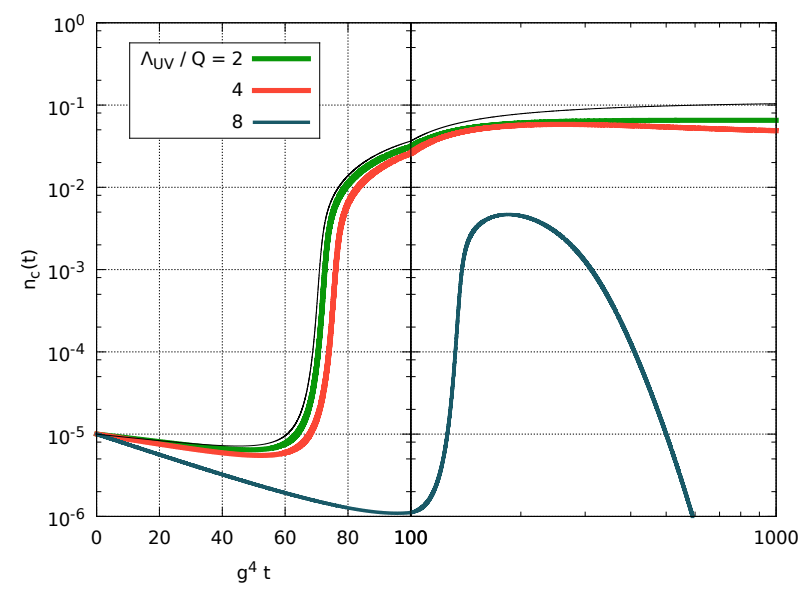

Figure 8: Time evolution of the density $n_{c}$ of condensed particles with the collision term in the classical approximation $\mathcal{C}^{1}$, for three values $\Lambda_{\mathrm{UV}} / Q=2,4$ and 8 of the UV cutoff. The thin black line is the solution of the unapproximated Boltzmann equation with $\Lambda_{\mathrm{UV}} / Q=8$.

of the cutoff, $\Lambda_{\mathrm{UV}} / Q=4$, for which the study of $n_{c}(t)$ indicated a qualitative agreement of the classical approximations with the exact quantum result.

We first show in the figure 9 the solution of the Boltzmann equation with an unapproximated collision term. Starting from a step-like distribution of the form (43), the distribution grows quickly at soft momenta, and also extends to momenta above $Q$. At times $g^{4} t \lesssim 70$, i.e. before the BEC transition, the distribution seems to remain bounded as $p / Q \rightarrow 0$. This soft behavior is drastically changed after the transition, since we now observe that $f(p) \sim p^{-2}$ as $p / Q \rightarrow 0$. In the soft sector, the distribution is well fitted by a functional form $T /\left(\omega_{\boldsymbol{p}}-m\right)$, which is indeed what one expects in the presence of a BEC, with a chemical potential equal to the mass of the particles. Already at $g^{4} t=100$, the soft sector of the distribution is already equilibrium-like, but with a temperature which is still too high compared to the final equilibrium temperature. The subsequent evolution is a slow decrease of this temperature, concomitant with a gradual extension of the tail of the distribution to larger momenta.

In the figures 10 and 11 , we show the values of $f(t, p)$ at the same times, respectively for the classical approximations $\mathcal{C}^{0}$ and $\mathcal{C}^{1}$. The first general remark is that the time-line of events is qualitatively the same as what we have described above in the quantum case. In fact, up to the BEC transition and shortly afterwards, the three evolutions are remarkably close, and the differences become more pronounced only at later times. The most obvious of these differences lie in the large momentum tail of the distribution, that behave very differently in the three cases considered (exact, approximations $\mathcal{C}^{0}$ and $\mathcal{C}^{1}$ ). This is of course expected, since the only common part of the collision term in these three 


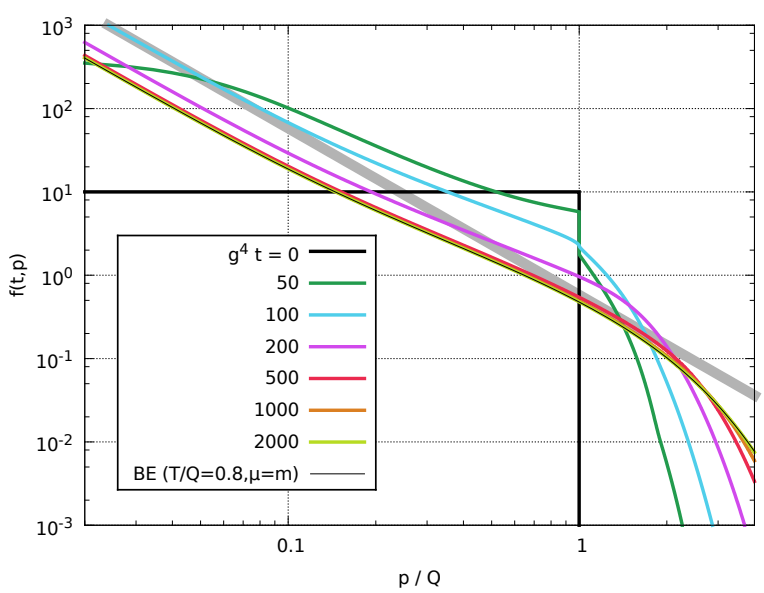

Figure 9: Particle distribution at various times, obtained with the unapproximated collision term. The UV cutoff is $\Lambda_{\mathrm{UV}} / Q=4$. The thin line is a fit with a Bose-Einstein distribution with chemical potential $\mu=m$. The gray band is a $p^{-2}$ power law.

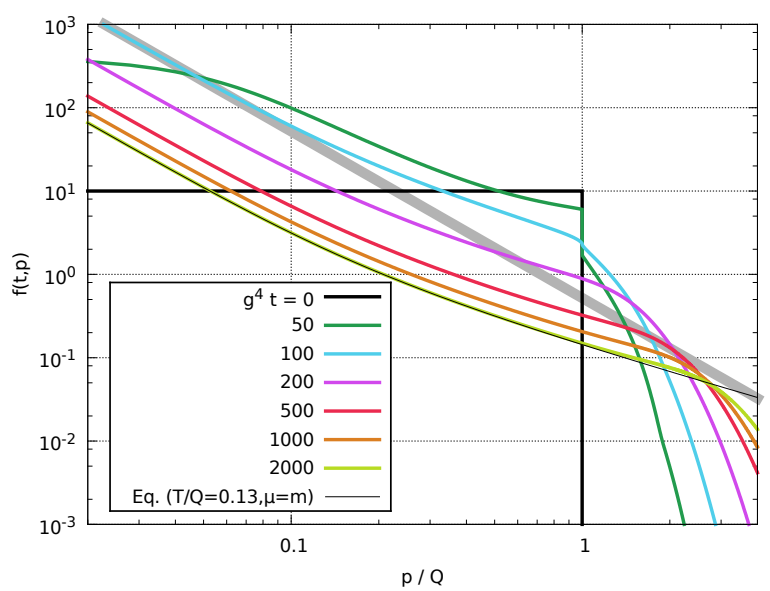

Figure 10: Particle distribution at various times, obtained with the collision term in the classical approximation $\mathcal{C}^{0}$. The UV cutoff is $\Lambda_{\mathrm{UV}} / Q=4$. The thin line is a fit with a classical equilibrium distribution of the form (33) with chemical potential $\mu=m$. The gray band is a $p^{-2}$ power law. 
situations is the term in $f^{3}$, which is not the leading one in the tail. Note also that these differences in the behavior of the tail are amplified when computing the total particle density or the energy density, since in these calculations the particle distribution is weighted by $p^{2}$ and $p^{3}$ respectively.

In the soft sector, the three computations show a behavior of the form $T /\left(\omega_{\boldsymbol{p}}-m\right)$ after the BEC transition, but the values of the effective temperature $T$ are somewhat different (smaller in the approximation $\mathcal{C}^{0}$ and larger in the approximation $\mathcal{C}^{1}$ ). Moreover, in the approximation $\mathcal{C}^{0}$, this effective temperature needs more time to settle to its equilibrium value.

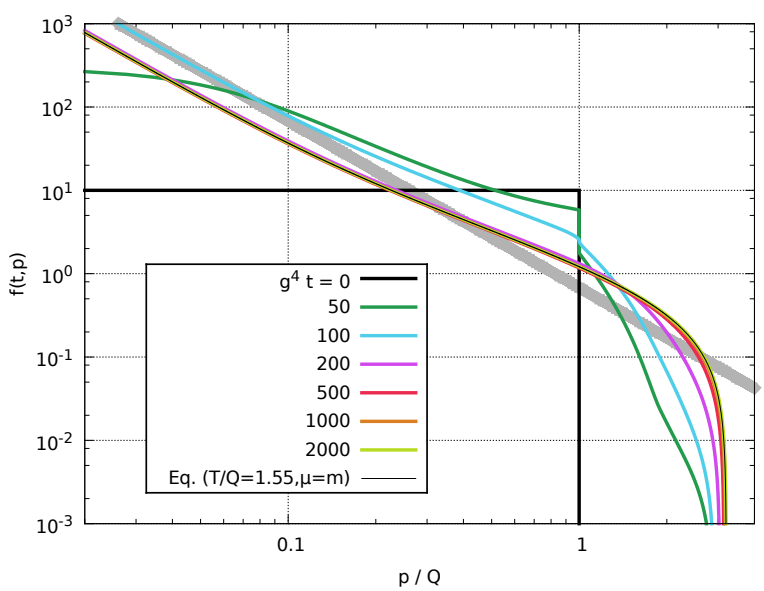

Figure 11: Particle distribution at various times, obtained with the collision term in the classical approximation $\mathcal{C}^{1}$. The UV cutoff is $\Lambda_{\mathrm{UV}} / Q=4$. The thin line is a fit with a classical equilibrium distribution of the form (35) with chemical potential $\mu=m$. The gray band is a $p^{-2}$ power law.

\section{Conclusions}

In this paper, we have explored various aspects of the classical approximation applied to the elastic collision term of the Boltzmann equation. The starting point of this study is the realization that the dependence of the effective temperature and chemical potential on the ultraviolet cutoff in classical statistical field simulations can be understood in a very simple way from kinetic theory arguments based on conservation laws. It therefore seemed natural to study more thoroughly the classical approximation of the Boltzmann equation, and in particular its sensitivity to the ultraviolet cutoff, as an alternative to the much more computationally demanding classical statistical simulations.

Our study is limited to the $2 \rightarrow 2$ collision term for scalar particles with a $\phi^{4}$ interaction. We have considered two versions of the classical. In the first 
one (denoted $\mathcal{C}^{0}$ ), one keeps only the cubic terms in the distribution function in the collision term. The CSA analogue of this approximation would be an initial ensemble of fields whose momentum spectrum falls like $\sqrt{f(0, \boldsymbol{p})}$. The second version of the classical approximation (denoted $\mathcal{C}^{1}$ ) amounts to replacing $f \rightarrow f+1 / 2$ in the collision term of the approximation $\mathcal{C}^{0}$. By doing this, one recovers the correct $f^{2}$ terms, but this also introduces some spurious terms that are linear in $f$. In the CSA, this approximation is analogous to an initial ensemble whose spectrum would include vacuum fluctuations, and thus behaves as $\sqrt{f(0, \boldsymbol{p})+1 / 2}$.

In the second version of the classical approximation, the collision term contains terms that are quadratic in the ultraviolet cutoff, even if the particle distribution has a compact support. These terms, that are closely related to some non-renormalizable contributions that were found in the CSA, make the solution of the Boltzmann approximation sensitive to the UV cutoff even at early times if the cutoff is much larger than the physical scales.

Without actually solving the Boltzmann equation, one can determine how its solution behaves at late times, solely from considerations based on conservation laws. The H-theorem can be generalized to the two classical approximations considered in this paper, provided that one modifies a bit the definition of entropy. By solving the conservation equations, we determined the cutoff dependence of the temperature and chemical potential that characterize the fixed point. In both approximations, these parameters are strongly cutoff dependent and become very different from the fixed point of the unapproximated Boltzmann equation if the cutoff is large.

Finally, we have solved numerically the Boltzmann equation, without any approximation and in the two versions of the classical approximation, for various values of the ultraviolet cutoff. As expected, the solutions of the unapproximated Boltzmann equation do not depend on the UV cutoff as soon as it is a few times larger than the physical scales. The situation is quite different in the classical approximation. The approximation $\mathcal{C}^{0}$ always leads to the formation of a Bose-Einstein condensate at large cutoff, regardless of whether the initial distribution is truly overoccupied or not. The opposite happens with the approximation $\mathcal{C}^{1}$, where no condensation takes place at large cutoff. However, if one keeps the cutoff a few times above the physical scales, it appeared that the two classical approximations reproduce, at least qualitatively, all the features of the exact solution. Given the fact that kinetic theory can account for the cutoff dependence of classical statistical computations, this analysis conversely suggests that CSA calculations done with a cutoff not exceeding 4-5 times the physical scales should be in good qualitative agreement with the unapproximated underlying quantum field theory.

\section{Acknowledgements}

We would like to thank J.-P. Blaizot, J. Liao and L. McLerran for useful discussions about this work. This work is support by the Agence Nationale de la 
Recherche project 11-BS04-015-01. N.T. is supported by JSPS Strategic Young Researcher Overseas Visits Program for Accelerating Brain Circulation (No. R2411).

\section{A $\quad \Sigma_{11}$ and $\Sigma_{12}$ at two loops (massless case)}

The collision term can be expressed in terms of the self-energies $\boldsymbol{\Sigma}_{11}$ and $\boldsymbol{\Sigma}_{12}$. For the $2 \rightarrow 2$ contribution, we need these self-energies at two loops. Since in the classical statistical approximation we neglect the vertex $\Gamma_{1112}$, they are given by a single Feynman diagram,

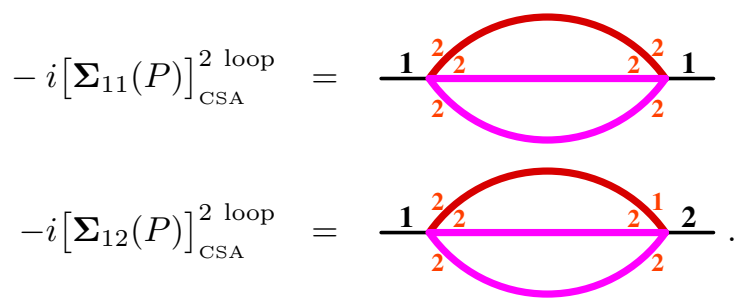

As we shall see, these graphs are ultraviolet divergent. We therefore regularize the integrals by limiting the 3 -momentum carried by the internal $G_{22}$ propagators,

$$
|\boldsymbol{p}| \leq \Lambda_{\mathrm{UV}} .
$$

The graphs in eqs. (44) contain as a subgraph the 1-loop $\Gamma_{1122} 4$-point function (highlighted in purple). In the massless case, we can use directly the result derived in the appendix B of ref. [18],

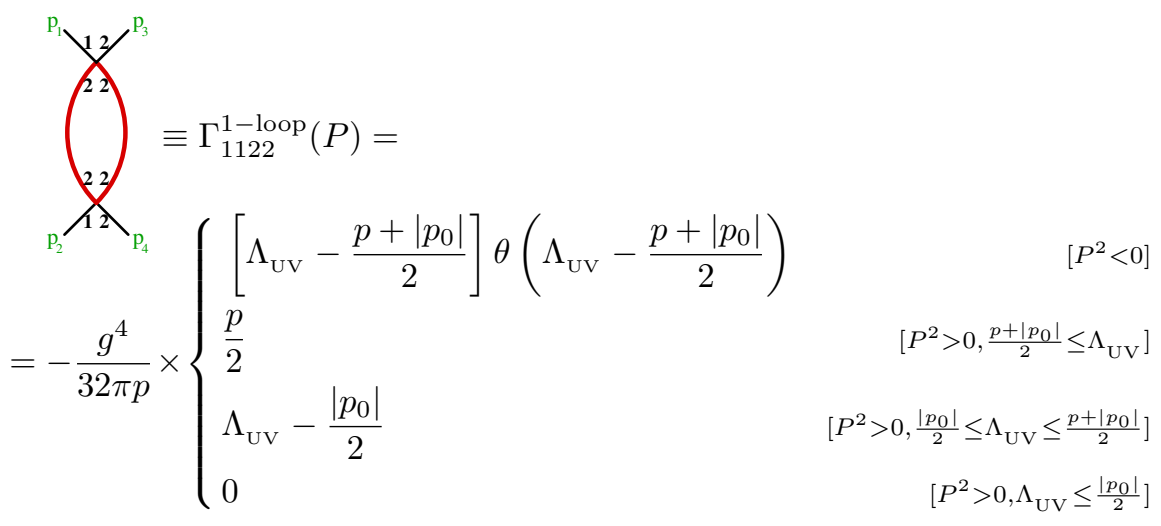

where $P^{\mu} \equiv p_{1}^{\mu}+p_{2}^{\mu}$ is the t-channel momentum. 
In terms of this subgraph, the expression for the required self-energies is

$$
\begin{aligned}
-i\left[\boldsymbol{\Sigma}_{11}(P)\right]_{\mathrm{CSA}}^{2 \text { loop }} & =\frac{g^{4}}{3} \int \frac{d^{4} K}{(2 \pi)^{4}} \pi \delta\left(K^{2}\right) \Gamma_{1122}^{1-\text { loop }}(P+K) \\
-i\left[\boldsymbol{\Sigma}_{12}(P)-\boldsymbol{\Sigma}_{21}(P)\right]_{\mathrm{CSA}}^{2 \text { loop }} & =g^{4} \int \frac{d^{4} K}{(2 \pi)^{4}} \pi \operatorname{sign}\left(k^{0}\right) \delta\left(K^{2}\right) \Gamma_{1122}^{1-\text { loop }}(P+K) .
\end{aligned}
$$

The two integrals are almost identical, except for the extra factor $\operatorname{sign}\left(k^{0}\right)$ in the second one. We can use the delta function $\delta\left(K^{2}\right)$ in order to perform the integral over $k^{0}$. The remain integrals over $k=|\boldsymbol{k}|$ and $\cos \theta$ (where $\theta$ is the angle between $\boldsymbol{k}$ and the external momentum $\boldsymbol{p}$ ) are elementary but require that one carefully splits the integration domain according to the various cases of eq. (46). For $P^{2}=0$ and $p^{0} \geq 0$, this leads to eqs. (19) and (20).

These CSA results contain ultraviolet divergences. However, note that $\Sigma_{11}$ at two loops is finite if calculated in the full theory :

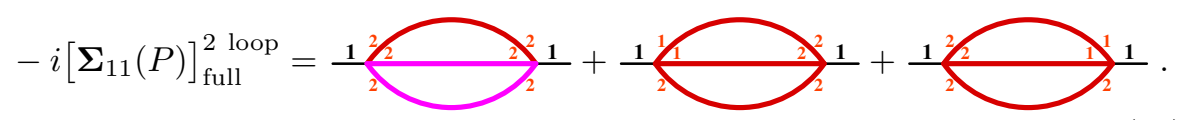

After some massaging of the integrands, the sum of these three graphs can be written as

$$
-i\left[\boldsymbol{\Sigma}_{11}(P)\right]_{\text {full }}^{2 \text { loop }}=-\frac{g^{4}}{12} \sum_{\epsilon= \pm}\left[\prod_{i=1}^{3} \int \frac{\mathrm{d}^{4} k_{i}}{(2 \pi)^{4}} \theta\left(\epsilon k_{i}^{0}\right) 2 \pi \delta\left(k_{i}^{2}\right)\right](2 \pi)^{4} \delta\left(\sum_{i} k_{i}-P\right)
$$

and it is easy to see that the two terms $\epsilon= \pm$ are separately ultraviolet finite. The finiteness of $\Sigma_{11}$ at two loops was expected from the renormalizability of the full theory ${ }^{18}$. One can check that $\boldsymbol{\Sigma}_{12}$ is also UV finite at two loops in the full theory.

\section{B Numerical evaluation of the collision term}

\section{B.1 Reduction to a 2-dimensional integral}

In the general case, the collision term is given by a 5-dimensional integral (three 3-dimensional phase-space integrals, minus 4 constraints coming from the conservation of energy and momentum). This is very costly to evaluate in a numerical study of the Boltzmann equation (this 5-dimensional integral would have to be calculated for every $\boldsymbol{p}$, at each timestep).

In order to simplify the numerics, we assume that the distribution is isotropic in momentum space. The scalar interactions being independent of the direction

\footnotetext{
${ }^{18}$ Intuitively, one should view $\Sigma_{11}$ as a sum of cut diagrams, in which the energy can flow only in one direction through the cut. At two loops, all the internal lines must be cut, so there cannot be any ultraviolet divergent subdiagram.
} 
of the particles involved in the scattering, this allows to separate completely the angular integrations from the integrations over the energies [33, 34]. We will illustrate this in the case of the unapproximated collision term (3), but the same procedure is applicable to the classical approximations considered in this paper.

Let us call $d \Omega_{p}$ the angular measure for the momentum $\boldsymbol{p}$,

$$
d \Omega_{\boldsymbol{p}}=\sin \theta_{\boldsymbol{p}} d \theta_{\boldsymbol{p}} d \phi_{\boldsymbol{p}} \quad, \quad \int d \Omega_{\boldsymbol{p}}=4 \pi,
$$

where $\phi_{\boldsymbol{p}}$ is the azimuthal angle and $\theta_{\boldsymbol{p}}$ the polar angle. Firstly, we can exploit the isotropy in order to write

$$
C_{\boldsymbol{p}}[f]=\frac{1}{4 \pi} \int d \Omega_{\boldsymbol{p}} C_{\boldsymbol{p}}[f] .
$$

The second trick is to write the delta function of momentum conservation as follows,

$$
(2 \pi)^{3} \delta\left(\boldsymbol{p}+\boldsymbol{k}-\boldsymbol{p}^{\prime}-\boldsymbol{k}^{\prime}\right)=\int d^{3} \boldsymbol{x} e^{i\left(\boldsymbol{p}+\boldsymbol{k}-\boldsymbol{p}^{\prime}-\boldsymbol{k}^{\prime}\right) \cdot \boldsymbol{x}},
$$

which allows to disentangle the angular integrations for the four momenta. Taking the direction of $\boldsymbol{x}$ as the polar axis, these angular integrals give

$$
\begin{aligned}
C_{\boldsymbol{p}}[f]= & \frac{g^{4}}{32 \pi^{4} p \omega_{\boldsymbol{p}}} \int d p^{\prime} d k d k^{\prime} \frac{p^{\prime} k k^{\prime}}{\omega_{\boldsymbol{p}^{\prime}} \omega_{\boldsymbol{k}} \omega_{\boldsymbol{k}^{\prime}}} \delta\left(\omega_{\boldsymbol{p}}+\omega_{\boldsymbol{k}}-\omega_{\boldsymbol{p}^{\prime}}-\omega_{\boldsymbol{k}^{\prime}}\right) \\
& \times \int_{0}^{\infty} \frac{d x}{x^{2}} \sin (p x) \sin (k x) \sin \left(p^{\prime} x\right) \sin \left(k^{\prime} x\right)\{f \cdots\},
\end{aligned}
$$

where $\{f \cdots\}$ denotes the combination of $f$ 's that enter in the collision term (only this factor needs to be modified if one wants to perform the same reduction in the case of the classical approximations). Using

$$
\int_{0}^{\infty} \frac{d x}{x^{2}} \sin (p x) \sin (k x) \sin \left(p^{\prime} x\right) \sin \left(k^{\prime} x\right)=\frac{\pi}{4} \operatorname{Min}\left(p p^{\prime} k k^{\prime}\right),
$$

we finally obtain ${ }^{19}$

$$
C_{\boldsymbol{p}}[f]=\frac{g^{4}}{128 \pi^{3} p \omega_{\boldsymbol{p}}} \int d \omega_{\boldsymbol{p}^{\prime}} d \omega_{\boldsymbol{k}^{\prime}} \operatorname{Min}\left(p p^{\prime} k k^{\prime}\right)\{f \cdots\},
$$

where the integration over the energy $\omega_{\boldsymbol{k}}$ has been eliminated thanks to the delta function. This form of the collision term is simple enough to be evaluated with high accuracy in a numerical resolution of the Boltzmann equation. In the figure 12, we have represented the allowed integration domain over the energies $\omega_{p^{\prime}}$ and $\omega_{\boldsymbol{k}^{\prime}}$, taking into account the fact that all the energies must be between $m$ and $\omega_{\Lambda} \equiv \sqrt{\Lambda_{\mathrm{UV}}^{2}+m^{2}}$. The domain can be divided into four subdomains, according to the value of $\operatorname{Min}\left(p p^{\prime} k k^{\prime}\right)$, that we have indicated inside the white disks.

\footnotetext{
${ }^{19}$ We have used $p d p=\omega d \omega$.
} 


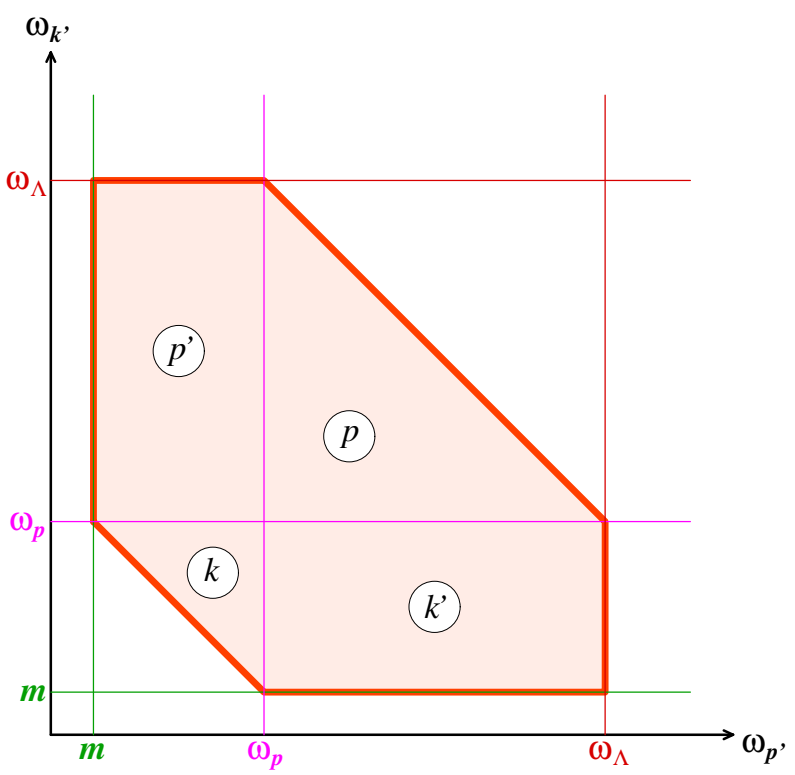

Figure 12: Allowed integration domain over the variables $\omega_{\boldsymbol{p}^{\prime}}$ and $\omega_{\boldsymbol{k}^{\prime}}$. The diagonal borders of this domain correspond to $\omega_{k}=m$ and $\omega_{k}=\omega_{\Lambda}$, respectively. The labels inside the white disks indicate the value of $\operatorname{Min}\left(p p^{\prime} k k^{\prime}\right)$ in the corresponding subdomain. 


\section{B.2 Number and energy conserving discretization}

In order to evaluate eq. (55), we must discretize momentum space, so that the 2-dimensional integral is replaced by a double discrete sum. For generic discretizations, the values of $p^{\prime}$ (obtained from $p, k, k^{\prime}$ by using energy conservation) will in general not fall on the grid points, and the value of $f\left(p^{\prime}\right)$ must therefore be obtained by interpolation, which may lead to some errors in the conservation laws.

A better discretization is to use linearly spaced points from $m$ to $\omega_{\Lambda}$ in the energy variables $\omega_{\boldsymbol{k}}$ and $\omega_{\boldsymbol{k}^{\prime}}$. If $\omega_{\boldsymbol{p}}$ is itself one of these points, then one is guaranteed that $\omega_{p^{\prime}}$ will also be on this grid, thereby eliminating the need for an interpolation. Let us denote these grid points $\omega_{0}=m, \omega_{1}, \cdots, \omega_{N} \equiv \omega_{\Lambda}$,

$$
\omega_{i}=m+i \Delta \quad, \quad \Delta \equiv \frac{\omega_{\Lambda}-m}{N} .
$$

We replace an integral over $\omega$ by the following quadrature formula ${ }^{20}$

$$
\int_{m}^{\Lambda} d \omega f(\omega) \rightarrow \Delta \sum_{i=1}^{N} w_{i} f\left(\omega_{i}\right)
$$

where the coefficients $w_{i}$ depend on the details of the quadrature formula.

In addition to avoiding the errors due to interpolations, the conservation of particle number and energy requires that the discretization exactly preserves the antisymmetry of the integrand if one swaps initial and final states, and its symmetry if one swaps the two particles of the initial state, or the two particles of the final state. In order to achieve this, it is useful to rewrite the 2-dimensional integral that enters in the collision term as follows

$$
\int d \omega_{\boldsymbol{p}^{\prime}} d \omega_{\boldsymbol{k}^{\prime}} \cdots=\int d \omega_{\boldsymbol{p}^{\prime}} d \omega_{\boldsymbol{k}^{\prime}} d \omega_{\boldsymbol{k}} \delta\left(\omega_{\boldsymbol{p}}+\omega_{\boldsymbol{k}}-\omega_{\boldsymbol{p}^{\prime}}-\omega_{\boldsymbol{k}^{\prime}}\right) \cdots
$$

After discretization, if $i$ is the discrete index corresponding to the energy $\omega_{\boldsymbol{p}}$, this integral becomes

$$
\Delta^{2} \sum_{j, k, l=1}^{N} w_{j} w_{k} w_{l} \delta_{i+j-k-l} \cdots=\Delta^{2} \sum_{k, l=1}^{N} w_{k} w_{l} w_{k+l-i} \cdots
$$

One can read from this formula the quadrature weights that should be used in the double sum that appears in the collision term, in order to preserve exactly ${ }^{21}$ all the symmetries that are necessary for the conservation laws.

The numerical results presented in this paper have been obtained by using the trapezoidal rule for the 1-dimensional integration :

$$
w_{1}=\frac{1}{2} \quad, \quad w_{2,3, \cdots, N-1}=1 \quad, \quad w_{N}=\frac{1}{2} .
$$

\footnotetext{
${ }^{20}$ We exclude the index $i=0$ from the sum, because it corresponds to $p=0$ where $f(p)$ may be singular. This point is handled by using the coupled equations for $f(p)$ and $n_{c}$ described in the section 3 , whose right hand side can be similarly reduced to 2-dimensional integrals.

${ }^{21}$ In practice, due to rounding errors, one finds that the particle number and the energy are conserved with machine accuracy.
} 


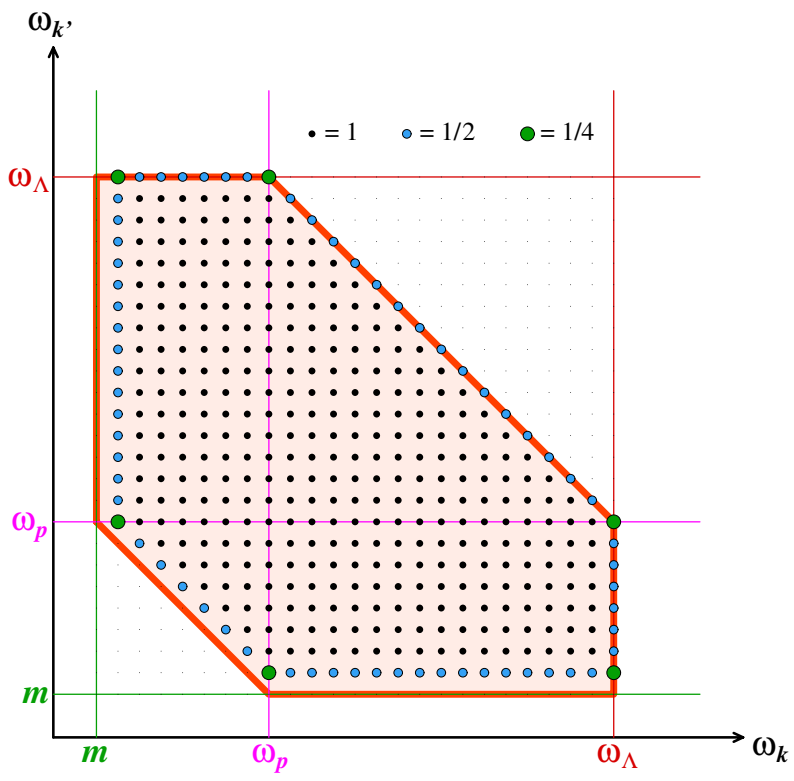

Figure 13: 2-dimensional quadrature weights for the trapezoidal rule, in the generic case where $\omega_{\boldsymbol{p}}$ is in the interior of the grid.
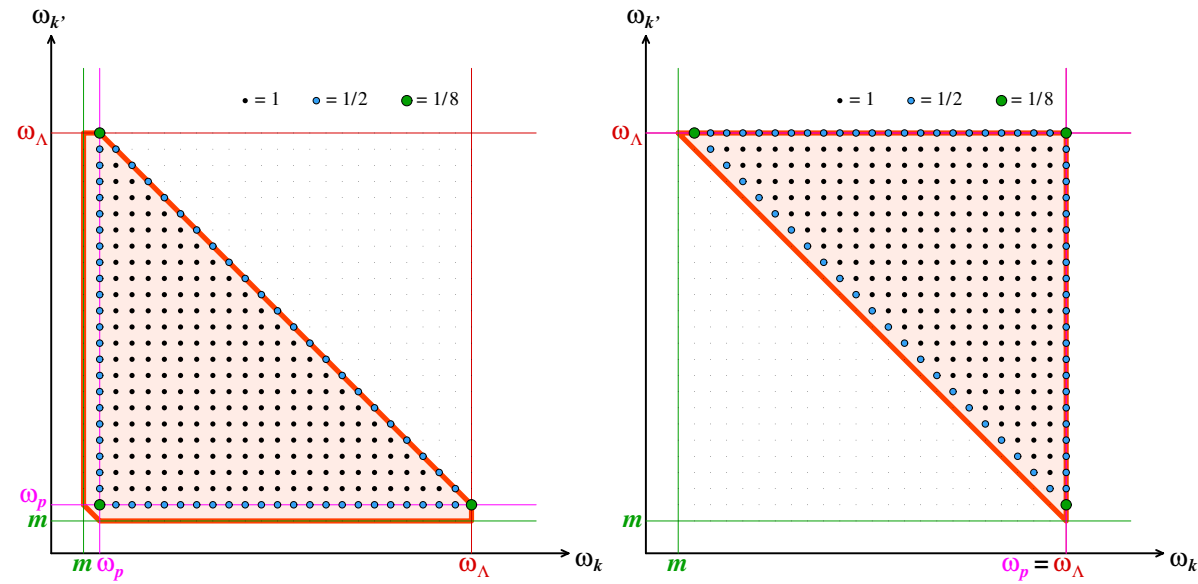

Figure 14: 2-dimensional quadrature weights for the trapezoidal rule, in the special cases where $\omega_{\boldsymbol{p}}$ is at one of the endpoints of the grid. 
For this choice, the 2-dimensional weights that lead to an exact conservation of particle number and energy are represented in the figures 13 (generic case) and 14 (when $\omega_{\boldsymbol{p}}$ is at one of the endpoints of the grid). Similar constructions can be done for higher order quadratures, such as Simpson's rule.

As a final note, let us stress that the computation time of the double sum in eq. (59) scales as $N^{2}$. At each time step, this must be repeated for each of the $N$ values of energy. Therefore, the total computation time scales as $N^{3}$ times the number of time steps. This strong dependence on $N$ puts a practical limit on the number of discrete values of the energy one can take (in the calculations presented in this paper, the largest value of $N$ that we used is $N=8000$.).

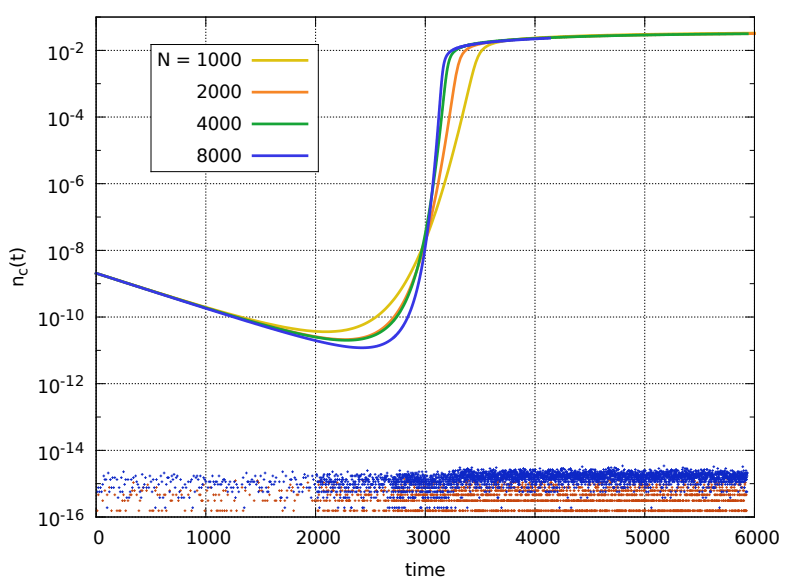

Figure 15: Numerical solution of eqs. (16) and (17) for an overoccupied initial condition. The curves show the time evolution of the density of condensed particles, $n_{c}(t)$, for various numbers $N$ of grid points. The scattered points at the bottom are the relative errors on the conservation of particle number (orange points) and energy (blue points).

In the figure 15, we show an example of the evolution of the density $n_{c}$ of condensed particles (for an overoccupied initial condition), obtained by solving the coupled equations (16) and (17) with the above discretization method. The scattered points are the relative error on the conservation of the number of particles (orange points) and of energy (blue points). The various curves correspond to different numbers of grid points in the discretization of the energy axis. Since we are considering only spatially homogeneous systems, the condensation transition should be a genuine discontinuity. However, in any discretization of momentum space, there will be a "smallest nonzero momentum", that in a sense plays the role of an inverse size of the system. One can see in the figure 15 that by increasing $N$ (i.e. increasing this system size), the transition seen in the numerical calculation becomes sharper. Note that in order to handle this fast transition, it is best to use an adaptative timestep, adjusted dynamically 
in order to limit the variation of $n_{c}$ during one step.

\section{Linear part of $C_{p}^{\mathcal{C}^{1}}[f]$}

In the classical approximation $\mathcal{C}^{1}$, the collision term is the sum of the full quantum collision term (that contains cubic and quadratic terms in $f$ ) and of an extra piece which is linear in $f$. After a tedious calculation, this additional term can be reduced in the massless case to a formula that contains at most 1-dimensional integrals,

$$
\begin{aligned}
C_{\boldsymbol{p}, \text { lin }}^{\mathcal{C}^{1}}[f]= & \frac{g^{4}}{512 p^{2} \pi^{3}}\left\{\frac{p f(p)}{6}\left(2 p^{2}-3 \Lambda_{\mathrm{UV}}^{2}\right)+\int_{0}^{p} d k k\left(2 \Lambda_{\mathrm{UV}}+k\right) f(k)\right. \\
& +p\left(2 \Lambda_{\mathrm{UV}}+p\right) \int_{p}^{\Lambda_{\mathrm{UV}}} d k f(k)+\int_{\Lambda_{\mathrm{UV}}-p}^{\Lambda_{\mathrm{UV}}} d k f(k)\left(p+k-\Lambda_{\mathrm{UV}}\right)^{2} \\
& \left.-3 p \int_{0}^{\Lambda_{\mathrm{UV}}} d k k f(k)\right\} .
\end{aligned}
$$

From this formula, one can check explicitly that this extra term also conserves particle number and energy, since it satisfies

$$
\begin{aligned}
& \int_{0}^{\Lambda_{\mathrm{UV}}} d p p^{2} C_{\boldsymbol{p}, \text { lin }}^{\mathcal{C}^{1}}[f]=0, \\
& \int_{0}^{\Lambda_{\mathrm{UV}}} d p p^{3} C_{\boldsymbol{p}, \operatorname{lin}}^{\mathcal{C}^{1}}[f]=0 .
\end{aligned}
$$

From eq. (61), it is also easy to extract the leading power in the UV cutoff. If we assume that the support of $f$ does not extend beyond $\Lambda_{\mathrm{UV}} / 2$ and that $p<\Lambda_{\mathrm{UV}} / 2$, we get

$$
C_{\boldsymbol{p}, \operatorname{lin}}^{\mathcal{C}^{1}}[f]=-\frac{g^{4} \Lambda_{\mathrm{UV}}^{2}}{1024 \pi^{3}} \frac{1}{p} f(\boldsymbol{p})+\cdots
$$

Note also that the linear part of the collision term can be written as

$$
C_{\boldsymbol{p}, \operatorname{lin}}^{\mathcal{C}^{1}}[f]=\frac{g^{4}}{512 p^{2} \pi^{3}} \int_{0}^{\Lambda_{\mathrm{UV}}} d p^{\prime} K\left(p, p^{\prime}\right) f\left(p^{\prime}\right),
$$

with

$$
K\left(p \cdot p^{\prime}\right) \equiv \frac{p\left(2 p^{2}-3 \Lambda_{\mathrm{UV}}^{2}\right)}{6} \delta\left(p-p^{\prime}\right)+\operatorname{Min}\left(p\left(2 \Lambda_{\mathrm{UV}}+p\right), p^{\prime}\left(2 \Lambda_{\mathrm{UV}}+p^{\prime}\right)\right)
$$


The kernel $K\left(p, p^{\prime}\right)$ is real symmetric, and therefore the linear part of the collision term, if viewed as a linear operator in the space of $f(p)$ 's, has a real spectrum and its eigenfunctions form an orthonormal basis. It is easy to check that this linear operator has a 2-dimensional null eigenspace, made of all the distributions of the form $f(p)=A+B\left(p / \Lambda_{\mathrm{UV}}\right)$. All the other eigenvalues are negative, which means that if we keep only this linear term in the Boltzmann equation, the solutions are attracted to this null space. Note that this fixed point is not realized in practice, because the terms in $f^{3}$ and $f^{2}$ become important before reaching it and modify the functional form of the allowed fixed points.

\section{Dependence of $n_{c}$ on the UV cutoff}

If a Bose-Einstein condensate exists in the system, one has $\mu=m$. As a result, $\widetilde{T}$ and $\widetilde{n}_{c}$ can be solved from eqs. (37), which take the form

$$
\begin{aligned}
& \widetilde{T}=6 \pi^{2}(\widetilde{\epsilon}-\widetilde{m} \tilde{n})+3 f(\widetilde{m})-\frac{1}{2} \widetilde{m}, \\
& \widetilde{n}_{c}=\widetilde{n}+\frac{1}{12 \pi^{2}}-\frac{\widetilde{T}}{2 \pi^{2}} \int_{0}^{1} \mathrm{~d} x \frac{x^{2}}{\sqrt{x^{2}+\widetilde{m}^{2}}-\widetilde{m}} .
\end{aligned}
$$

In the massless limit $m \rightarrow 0$, one has

$$
T=\frac{3}{8} \Lambda_{\mathrm{UV}}+\frac{6 \pi^{2} \epsilon}{\Lambda_{\mathrm{UV}}^{3}}, \quad n_{c}=n-\frac{3 \epsilon}{2 \Lambda_{\mathrm{UV}}}-\frac{\Lambda_{\mathrm{UV}}^{3}}{96 \pi^{2}} .
$$

For the initial condition (43), the condition for the existence of a Bose-Einstein condensate is given by

$$
\frac{\Lambda_{\mathrm{UV}}}{Q}<2\left[2 f_{0}\left(1-\frac{9}{8} \frac{Q}{\Lambda_{\mathrm{UV}}}\right)\right]^{\frac{1}{3}} .
$$

For any $f_{0}$, there is always a limiting value of $\Lambda_{\mathrm{UV}}$ above which there cannot be a BEC.

Similarly, in the case of the classical approximation $\mathcal{C}^{0}$ one has

$$
T=\frac{6 \pi^{2} \epsilon}{\Lambda_{\mathrm{UV}}^{3}}, \quad n_{c}=n-\frac{3 \epsilon}{2 \Lambda_{\mathrm{UV}}},
$$

and the condition for the existence of a Bose-Einstein condensate is given by

$$
\Lambda_{\mathrm{UV}}>\frac{3 \epsilon}{2 n}=\frac{9}{8} Q
$$

For the approximation $\mathcal{C}^{0}$, the situation is reversed: at large enough cutoff, a BEC would always be present. 


\section{References}

[1] T. Lappi, L.D. McLerran, Nucl. Phys. A 772, 200 (2006).

[2] F. Gelis, E. Iancu, J. Jalilian-Marian, R. Venugopalan, Ann. Rev. Part. Nucl. Sci. 60, 463 (2010).

[3] K. Fukushima, F. Gelis, Nucl. Phys. A 874, 108 (2012).

[4] F. Gelis, R. Venugopalan, Nucl. Phys. A 776, 135 (2006).

[5] F. Gelis, T. Lappi, R. Venugopalan, Phys. Rev. D 78, 054019 (2008).

[6] P. Romatschke, R. Venugopalan, Phys. Rev. D 74, 045011 (2006).

[7] K. Dusling, T. Epelbaum, F. Gelis, R. Venugopalan, Nucl. Phys. A 850, 69 (2011).

[8] T. Epelbaum, F. Gelis, Nucl. Phys. A 872, 210 (2011).

[9] T. Epelbaum, F. Gelis, Phys. Rev. Lett. 111, 232301 (2013).

[10] J. Berges, K. Boguslavski, S. Schlichting, Phys. Rev. D 85, 076005 (2012).

[11] J. Berges, S. Schlichting, D. Sexty, Phys. Rev. D 86, 074006 (2012).

[12] J. Berges, K. Boguslavski, S. Schlichting, R. Venugopalan, Phys. Rev. D 89, 074011 (2014).

[13] J. Berges, K. Boguslavski, S. Schlichting, R. Venugopalan, Phys. Rev. D 89, 114007 (2014).

[14] J. Berges, K. Boguslavski, S. Schlichting, R. Venugopalan, JHEP 1405, 054 (2014).

[15] K. Fukushima, F. Gelis, L. McLerran, Nucl. Phys. A 786, 107 (2007).

[16] K. Dusling, F. Gelis, R. Venugopalan, Nucl. Phys. A 872, 161 (2011).

[17] T. Epelbaum, F. Gelis, Phys. Rev. D 88, 085015 (2013).

[18] T. Epelbaum, F. Gelis, B. Wu, arXiv:1402.0115, to appear in PRD.

[19] M.C. Abraao York, A. Kurkela, E. Lu, G.D. Moore, Phys. Rev. D 89, 074036 (2014).

[20] A. Kurkela, E. Lu, arXiv:1405.6318.

[21] P. Arnold, G.D. Moore, L.G. Yaffe, JHEP 0301, 030 (2003).

[22] A.H. Mueller, D.T. Son, Phys. Lett. B 582, 279 (2004).

[23] S. Jeon, Phys. Rev. C 72, 014907 (2005). 
[24] J. Schwinger, J. Math. Phys. 2, 407 (1961).

[25] L.V. Keldysh, Sov. Phys. JETP 20, 1018 (1964).

[26] M.A. van Eijck, R. Kobes, Ch.G. van Weert, Phys. Rev. D 50, 4097 (1994).

[27] S. Jeon, Annals Phys. 340, 119 (2014).

[28] J.P. Blaizot, J. Liao, L.D. McLerran, Nucl. Phys. A 920, 58 (2013).

[29] J.P. Blaizot, B. Wu, L. Yan, arXiv:1402.5049.

[30] R. Lacaze, P. Lallemand, Y. Pomeau, S. Rica, Physica D 152, 779 (2001).

[31] R. Kubo, J. Phys. Soc. Japan 12, 570 (1957).

[32] P.C. Martin, J. Schwinger, Phys. Rev. 115, 1342 (1959).

[33] D.V. Semikoz, I.I. Tkachev, Phys. Rev. Lett. 74, 3093 (1995).

[34] D.V. Semikoz, I.I. Tkachev, Phys. Rev. D 55, 489 (1997). 